\title{
LI-YAU HARNACK ESTIMATES FOR A HEAT-TYPE EQUATION UNDER THE GEOMETRIC FLOW
}

\author{
YI LI AND XIAORUI ZHU
}

\begin{abstract}
In this paper, we consider the gradient estimates for a postive solution of the nonlinear parabolic equation $\partial_{t} u=\Delta_{t} u+h u^{p}$ on a Riemannian manifold whose metrics evolve under the geometric flow $\partial_{t} g(t)=-2 S_{g(t)}$. To obtain these estimate, we introduce a quantity $\boldsymbol{S}$ along the flow which measures whether the tensor $S_{i j}$ satisfies the second contracted Bianchi identity. Under conditions on $\operatorname{Ric}_{g(t)}, S_{g(t)}$, and $\underline{\boldsymbol{S}}$, we obtain the gradient estimates.
\end{abstract}

\section{INTRODUCTION}

We are continuous to consider the gradient estimates for nonlinear partial differential equations after our previous works $[6,11,12]$. Let $(M, g(t))_{t \in[0, T]}$ be a complete solution to the geomtric flow

$$
\partial_{t} g(t)=-2 S_{g(t)}, \quad t \in[0, T] .
$$

on a complete and noncompact $n$-dimensional manifold $M$ and consider a positive function $u=u(x, t)$ defined on $M \times[0, T]$ solving the equation

$$
\partial_{t} u=\Delta_{t} u+h u^{p}, \quad t \in[0, T],
$$

where $\Delta_{t}$ stands for the Laplacian of $g(t), h$ is a function defined on $M \times[0, T]$ which is $C^{2}$ in $x$ and $C^{1}$ in $t$, and $p$ is a positive constant. When metrics are fixed, the study on the gradient estimates of (1.2) arose from [4]. If $h=0$, Sun [9] derived the gradient estimates and the Harnack inequalities for the positive solutions of the linear parabolic equation $\partial_{t} u=\Delta_{t} u$ under the geometric flow. In this paper, we consider the general case for the nonlinear parabolic equation. Notice that the $\Delta_{t}$ depends on the parameter $t$, and we should study the equation (1.2) coupled with the geometric flow (1.1). The formula (1.1) provides us with additional information about the coefficients of the operator $\Delta_{t}$ appearing in (1.2) but is itself fully independent of (1.2).

We introduce notions used throughout this paper. Let $B_{\rho, T}=\{(x, t) \in M \times$ $\left.[0, T]: \operatorname{dist}_{g(t)}\left(x, x_{0}\right)<\rho\right\}$, where $\operatorname{dist}_{g(t)}\left(x, x_{0}\right)$ denotes the distance between $x$ to a fixed point $x_{0}$ with respect to $g(t) \cdot \nabla_{g(t)}$ and $|\cdot|_{g(t)}$ stand for the Levi-Civita connection and norm with respect to $g(t)$ respectively. Set

$$
\underline{S}_{g}(t):=\operatorname{div}_{g(t)} S_{g(t)}-\frac{1}{2} \nabla_{g(t)}\left(\operatorname{tr}_{g(t)} S_{g(t)}\right) .
$$

2010 Mathematics Subject Classification. Primary 53C44.

Key words and phrases. Nonlinear parabolic equation, Harnack estimate, geometric flow.

Yi Li is partially supported by the Fonds National de la Recherche Luxembourg (FNR) unde the OPEN scheme (project GEOMREV O14/7628746). Xiaorui Zhu is partially supported by Zhejiang Province Natural Science Foundation of China (grant) No. Q14A010002. 
Locally, one has

$$
\underline{\boldsymbol{S}}_{i}=\nabla^{j} S_{i j}-\frac{1}{2} \nabla_{i}\left(\operatorname{tr}_{g(t)} S_{g(t)}\right) .
$$

For example, if $S_{i j}=R_{i j}$, that is, (1.1) is the Ricci flow, we arrive at

$$
\underline{\boldsymbol{S}}_{i}=\nabla^{j} R_{i j}-\frac{1}{2} \nabla_{i} R_{g(t)}=\frac{1}{2} \nabla_{i} R_{g(t)}-\frac{1}{2} \nabla_{i} R_{g(t)}=0
$$

by the second contracted Bianchi identity. Thus, the quantity $\underline{\boldsymbol{S}}_{g(t)}$ measures whether $S_{i j}$ satisfies the second contracted Bianchi identity.

Theorem 1.1. Suppose that $(M, g(t))_{t \in[0, T]}$ is a solution to the geometric flow (1.1) on $M$ with $-K_{1} g(t) \leq S_{g(t)} \leq K_{2} g(t),-K_{3} g(t) \leq \operatorname{Ric}_{g(t)}-S_{g(t)} \leq K_{3} g(t)$, and $\left|\underline{\boldsymbol{S}}_{g(t)}\right|_{g(t)} \leq K_{4}$ for some $K_{1}, K_{2}, K_{3}, K_{4}>0$ on $B_{2 R, T}$, with $\bar{K}:=\max \left\{K_{1}, K_{2}\right\}$. Let $h(x, t)$ be a function defined on $M \times[0, T]$ which is $C^{2}$ in $x$ and $C^{1}$ in $t$, satisfying $\Delta_{g(t)} h \geq-\theta$ and $\left|\nabla_{g(t)} h\right|_{g(t)} \leq \gamma$ on $B_{2 R, T} \times[0, T]$ for some nonnegative constants $\theta$ and $\gamma$. If $u(x, t)$ is a positive smooth solution of (1.2) on $M \times[0, T]$, then

(i) for $0<p<1$, we have

$$
\begin{aligned}
\frac{\left|\nabla_{g(t)} u\right|_{g(t)}^{2}}{u^{2}}+\frac{h}{p} u^{p-1}-\frac{1}{p} \frac{u_{t}}{u} \leq & \frac{C_{1}}{p^{2} t}+\frac{n(1-p)}{p^{2}} M_{1} M_{2}+\frac{n\left[3 K_{1}+2\left(K_{3}+K_{4}\right) p\right]}{2 p^{2}(1-p)} \\
& +\frac{C_{1}}{p^{2}}\left(\frac{1}{R^{2}}+\frac{\sqrt{K_{1}+K_{3}}}{R}+\bar{K}+\frac{n}{p(1-p)}\right) \\
& +\left(\frac{n}{p}\right)^{3 / 2} \sqrt{\theta M_{2}}+\frac{\sqrt{n / K_{1}}}{p} \gamma M_{2}+\frac{n}{p^{2}} \sqrt{\frac{K_{4}}{2 n}}
\end{aligned}
$$

where $C_{1}$ is a positive constant depending only on $n$ and

$$
M_{1}:=\max _{B_{2 R, T}} h_{-}, \quad M_{2}:=\max _{B_{2 R, T}} u^{p-1}, \quad h_{-}:=\max (-h, 0) .
$$

(ii) for $p \geq 1$, we have

$$
\begin{aligned}
\frac{\left|\nabla_{g(t)} u\right|_{g(t)}^{2}}{u^{2}}+\frac{h}{p} u^{p-1}-\frac{1}{p} \frac{u_{t}}{t} \leq & \frac{k^{2} C_{2}}{p^{2} t}+\frac{n k^{2}(p-1)}{p^{2}} M_{4} M_{5}+\frac{k^{3} n}{k-p} M_{3} M_{4} \\
& +\frac{k^{2} C_{2}}{p^{2}}\left(\frac{1}{R^{2}}+\frac{\sqrt{K_{1}+K_{3}}}{R}+\bar{K}+\frac{k^{2} n}{p(k-p)}\right) \\
& +\frac{2 k^{3} n}{(k-p) p^{2}}\left[K_{1}+\frac{p}{k}\left(K_{3}+K_{4}\right)\right]+\frac{k^{2} \sqrt{n} \gamma}{p} M_{4} \\
& +\left(\frac{k n}{p}\right)^{3 / 2} \sqrt{\theta M_{4}}+\frac{k^{2} n}{p^{2}}\left(\bar{K}+\sqrt{\frac{K_{4}}{2 n}}\right),
\end{aligned}
$$

where $k>p, C_{2}$ is a positive constant depending only on $n$ and

$$
M_{3}:=\max _{B_{2 R, T}} h_{-}, \quad M_{4}:=\max _{B_{2 R, T}} u^{p-1}, \quad M_{5}:=\max _{B_{2 R, T}} h .
$$

As an immediate consequence of the above theorem we have

Theorem 1.2. Suppose that $(M, g(t))_{t \in[0, T]}$ is a solution to the geometric flow (1.1) on $M$. Let $h(x, t)$ be a function defined on $M \times[0, T]$ which is $C^{2}$ in $x$ and $C^{1}$ in $t$. 
(i) For $0<p<1$, assume that $h \geq 0$, $\left|\nabla_{g(t)} h\right|_{g(t)} \leq \gamma, \Delta_{g(t)} h \geq 0$ along the geometric flow with $-K_{1} g(t) \leq S_{g(t)} \leq K_{2} g(t),-K_{3} g(t) \leq \mathrm{Ric}_{g(t)}-S_{g(t)} \leq$ $K_{3} g(t),\left|\underline{\boldsymbol{S}}_{g(t)}\right|_{g(t)} \leq K_{4}$ for some positive constants $\gamma, K_{1}, K_{2}, K_{3}, K_{4}$ with $\bar{K}:=$ $\max \left\{K_{1}, K_{2}\right\}$, along the geometric flow. If $u$ is a smooth positive function satisfying the nonlinear parabolic equation (1.2), then

$$
\begin{aligned}
\frac{\left|\nabla_{g(t)} u\right|_{g(t)}^{2}}{u^{2}}+\frac{h}{p} u^{p-1}-\frac{1}{p} \frac{u_{t}}{u} \leq & \frac{C_{1}}{p^{2} t}+\frac{C_{1}}{p^{3}(1-p)}+\frac{C_{1}}{p^{2}} \bar{K}+\frac{2 n K_{1}}{p^{2}(1-p)} \\
& +\frac{\sqrt{n / K_{1}}}{p} \gamma M+\frac{n}{p^{2}} \sqrt{\frac{K_{4}}{2 n}}+\frac{n\left(K_{3}+K_{4}\right)}{p(1-p)}
\end{aligned}
$$

for some positive constant $C_{1}$ depending only on $n$, where $M:=\max _{M \times[0, T]} u^{p-1}$.

(ii) For $p=1$, assume that $-K_{1} g(t) \leq S_{g(t)} \leq K_{2} g(t),-K_{3} g(t) \leq \mathrm{Ric}_{g(t)}-$ $S_{g(t)} \leq K_{3} g(t),\left|\underline{\boldsymbol{S}}_{g(t)}\right|_{g(t)} \leq K_{4}$ for some positive constants $K_{1}, K_{2}, K_{3}, K_{4}$ with $\bar{K}:=\max \left\{K_{1}, K_{2}\right\}, h \geq 0, \Delta_{g(t)} h \geq-\theta$ ( $\theta$ is nonnegative $)$, and $\left|\nabla_{g(t)} h\right|_{g(t)} \leq \gamma$ ( $\gamma$ is nonnegative), along the geometric flow. If $u$ is a smooth positive function satisfying the nonlinear parabolic equation (1.2), then

$$
\frac{\left|\nabla_{g(t)} u\right|_{g(t)}^{2}}{u^{2}}+h-\frac{u_{t}}{u} \leq \frac{C_{2}}{t}+C_{2}\left(1+K_{1}+K_{2}+K_{3}+K_{4}+\bar{K}+\gamma+\sqrt{\theta}\right)
$$

for some positive constant $C_{2}$ depending only on $n$.

(iii) For $p>1$, assume that $-K_{1} g(t) \leq S_{g(t)} \leq K_{2} g(t),-K_{3} g(t) \leq \mathrm{Ric}_{g(t)}-$ $S_{g(t)} \leq K_{3} g(t),\left|\underline{\boldsymbol{S}}_{g(t)}\right|_{g(t)} \leq K_{4}$ for some positive constants $\gamma, K_{1}, K_{2}, K_{3}, K_{4}$ with $\bar{K}:=\max \left\{K_{1}, K_{2}\right\} . \Delta_{g(t)} h \geq-\theta,\left|\nabla_{g(t)} h\right|_{g(t)} \leq \gamma$, and $-k_{1} \leq h \leq k_{2}$, where $\theta, \gamma, k_{1}, k_{2}>0$, along the geometric flow. If $u$ is a bounded smooth positive function satisfying the nonlinear parabolic equation (1.2), then

$$
\begin{aligned}
\frac{\left|\nabla_{g(t)} u\right|_{g(t)}^{2}}{u^{2}}+\frac{h}{p} u^{p-1}-\frac{1}{p} \frac{u_{t}}{u} \leq & \left(\frac{k}{p}\right)^{2} \frac{C_{3}}{t}+\left(\frac{k}{p}\right)^{3} \frac{k}{k-p} C_{3}+\left(\frac{k}{p}\right)^{2} C_{3}(\bar{K}+ \\
& \left.+\frac{k}{k-p}\left(K_{1}+K_{3}+K_{4}\right)+\sqrt{\frac{K_{4}}{2 n}}\right) \\
& +\left(\frac{k}{p}\right)^{2} n(p-1) k_{2} M+\frac{k^{3} n}{k-p} k_{1} M \\
& +\frac{k^{2} \sqrt{n}}{p} \gamma M+\left(\frac{k n}{p}\right)^{3 / 2} \sqrt{\theta M},
\end{aligned}
$$

for some positive constant $C_{3}$ depending only on $n$, where $M:=\max _{M \times[0, T]} u^{p-1}$ and $k>p$. In particular, taking $k=2 p$, we get

$$
\begin{aligned}
\frac{\left|\nabla_{g(t)} u\right|_{g(t)}^{2}}{u^{2}}+\frac{h}{p} u^{p-1}-\frac{1}{p} \frac{u_{t}}{u} \leq & \frac{C_{4}}{t}+C_{5}\left(1+K_{1}+K_{2}+K_{3}+K_{4}+\bar{K}\right) \\
& +C_{4} p^{2}\left[\left(k_{1}+k_{2}\right) M+\gamma M+\sqrt{\theta M}\right],
\end{aligned}
$$

for some positive constant $C_{4}$ depending only on $n$.

Another type of Harnack inequality is the following

Theorem 1.3. Suppose that $(M, g(t))_{t \in[0, T]}$ is a solution to the geometric flow (1.1) on $M$, satisfying $-K_{1} g(t) \leq S_{g(t)} \leq K_{2} g(t),-K_{3} g(t) \leq \operatorname{Ric}_{g(t)}-S_{g(t)} \leq K_{3} g(t)$, $\left|\nabla_{g(t)} \underline{\boldsymbol{S}}_{g(t)}\right|_{g(t)} \leq K_{4}$, for some $K_{1}, K_{2}, K_{3}, K_{4}>0$, with $\bar{K}:=\max \left\{K_{1}, K_{2}\right\}$. Let 
$h(x, t)$ be a nonnegative function defined on $M \times[0, T]$ which is $C^{2}$ in $x$ and $C^{1}$ in $t, \Delta_{g(t)} h+h_{t}-2 C_{n, p} p \frac{\left|\nabla_{g(t)} h\right|_{g(t)}^{2}}{h} \geq 0$ on $M \times[0, T]$ (where $C_{n, p}=\frac{p}{p-1}$ if $p>1$ and $C_{n, p}=n$ if $\left.p \leq 1\right)$, and $0<p \leq \frac{2 n}{2 n-1}(n \geq 3)$. If $u$ is a positive solution of (1.2), then

$$
\begin{aligned}
\frac{\left|\nabla_{g(t)} u\right|_{g(t)}^{2}+\frac{h}{p} u^{p-1}-\frac{2}{p} \frac{u_{t}}{u} \leq}{u^{2}} & \frac{C}{p^{2} t}+\frac{8 n}{p^{2}} \bar{K}+\frac{8 n}{p^{2}} \sqrt{\frac{2 n}{p(2-p)} K_{1}} \\
& +\frac{4 n}{p(2-p)}\left(K_{1}+K_{3}+K_{4}\right)+\frac{1}{p^{2}} \sqrt{8 n K_{4}}
\end{aligned}
$$

for some positive constant $C$ depending only on $n$.

This theorem has three important consequences.

Corollary 1.4. Suppose that $(M, g(t))_{t \in[0, T]}$ is a solution to the geometric flow (1.1) on $M$, satisfying $0 \leq S_{g(t)} \leq K_{2} g(t),-K_{3} \leq \mathrm{Ric}_{g(t)}-S_{g(t)} \leq K_{3} g(t)$, $\left|\nabla_{g(t)} \underline{\boldsymbol{S}}_{g(t)}\right|_{g(t)} \leq K_{4}$, for some positive constants $\bar{K}_{2}, K_{3}, K_{4}$. Let $h(x, t)$ be a nonnegative function defined on $M \times[0, T]$ which is $C^{2}$ in $x$ and $C^{1}$ in $t, \Delta_{g(t)} h+$ $h_{t}-2 C_{n, p} p \frac{\left|\nabla_{g(t)} h\right|_{g(t)}^{2}}{h} \geq 0$ on $M \times[0, T]$ (where $C_{n, p}=\frac{p}{p-1}$ if $p>1$ and $C_{n, p}=n$ if $p \leq 1)$, and $0<p \leq \frac{2 n}{2 n-1}(n \geq 3)$. If $u$ is a positive solution of (1.2), then

$$
\frac{\left|\nabla_{g(t)} u\right|_{g(t)}^{2}}{u^{2}}+\frac{h}{p} u^{p-1}-\frac{2}{p} \frac{u_{t}}{t} \leq \frac{C}{p^{2} t}+\frac{8 n}{p^{2}} K_{2}+\frac{4 n}{p(2-p)}\left(K_{3}+K_{4}\right)+\frac{1}{p^{2}} \sqrt{8 n K_{4}}
$$

for some positive constant $C$ depending only on $n$.

Corollary 1.5. Suppose that $(M, g(t))_{t \in[0, T]}$ is a solution to the geometric flow (1.1) on $M$, satisfying $-K_{1} g(t) \leq S_{g(t)} \leq K_{2} g(t),-K_{3} g(t) \leq \operatorname{Ric}_{g(t)}-S_{g(t)} \leq$ $K_{3} g(t),\left|\nabla_{g(t)} \underline{\boldsymbol{S}}_{g(t)}\right|_{g(t)} \leq K_{4}$, for some $K_{1}, K_{2}, K_{3}, K_{4}>0$, with $\bar{K}:=\max \left\{K_{1}, K_{2}\right\}$. Let $h(x, t)$ be a nonnegative function defined on $M \times[0, T]$ which is $C^{2}$ in $x$ and $C^{1}$ in $t, \Delta_{g(t)} h+h_{t}-2 C_{n, p} p \frac{\left|\nabla_{g(t)} h\right|_{g(t)}^{2}}{h} \geq 0$ on $M \times[0, T]$ (where $C_{n, p}=\frac{p}{p-1}$ if $p>1$ and $C_{n, p}=n$ if $\left.p \leq 1\right)$, and $0<p \leq \frac{2 n}{2 n-1}(n \geq 3)$. If $u$ is a positive solution of (1.2), then

$$
\begin{aligned}
\frac{u\left(x_{2}, t_{2}\right)}{u\left(x_{1}, t_{1}\right)} \geq & \left(\frac{t_{2}}{t_{1}}\right)^{-C / p} \exp \left[-\frac{1}{2 p} \min _{\gamma \in \Theta\left(x_{1}, t_{1}, x_{2}, t_{2}\right)} \int_{t_{1}}^{t_{2}}|\dot{\gamma}(t)|_{g(t)}^{2} d t-2 n\left(t_{2}-t_{1}\right)\right. \\
& \left.\left(\frac{1}{p} \bar{K}+\frac{2}{p} \sqrt{\frac{2 n}{p(2-p)} K_{1}}+\frac{1}{2-p}\left(K_{1}+K_{3}+K_{4}\right)+\frac{1}{p} \sqrt{2 n K_{4}}\right)\right]
\end{aligned}
$$

for some positive constant $C$ depending only on $n$, where $\left(x_{1}, t_{1}\right),\left(x_{2}, t_{2}\right) \in M \times$ $[0, T]$ with $t_{1}<t_{2}$.

When $K_{1}=0$, we have the following

Corollary 1.6. Suppose that $(M, g(t))_{t \in[0, T]}$ is a solution to the geometric flow (1.1) on $M$, satisfying $0 \leq S_{g(t)} \leq K_{2} g(t),-K_{3} g(t) \leq \operatorname{Ric}_{g(t)}-S_{g(t)} \leq K_{3} g(t)$, $\left|\nabla_{g(t)} \underline{\boldsymbol{S}}_{g(t)}\right|_{g(t)} \leq K_{4}$, for some $K_{2}, K_{3}, K_{4}>0$. Let $h(x, t)$ be a nonnegative function defined on $M \times[0, T]$ which is $C^{2}$ in $x$ and $C^{1}$ in $t, \Delta_{g(t)} h+h_{t}-$ $2 C_{n, p} p \frac{\left|\nabla_{g(t)} h\right|_{g(t)}^{2}}{h} \geq 0$ on $M \times[0, T]$ (where $C_{n, p}=\frac{p}{p-1}$ if $p>1$ and $C_{n, p}=n$ 
if $p \leq 1)$, and $0<p \leq \frac{2 n}{2 n-1}(n \geq 3)$. If $u$ is a positive solution of (1.2), then

$$
\begin{aligned}
\frac{u\left(x_{2}, t_{2}\right)}{u\left(x_{1}, t_{1}\right)} \geq & \left(\frac{t_{2}}{t_{1}}\right)^{-C / p} \exp \left[-\frac{1}{2 p} \min _{\gamma \in \Theta\left(x_{1}, t_{1}, x_{2}, t_{2}\right)} \int_{t_{1}}^{t_{2}}|\dot{\gamma}(t)|_{g(t)}^{2} d t\right. \\
& \left.-2 n\left(t_{2}-t_{1}\right)\left(\frac{K_{2}}{p}+\frac{K_{3}+K_{4}}{2-p}+\frac{\sqrt{2 n K_{4}}}{p}\right)\right]
\end{aligned}
$$

for some positive constant $C$ depending only on $n$, where $\left(x_{1}, t_{1}\right),\left(x_{2}, t_{2}\right) \in M \times$ $[0, T]$ with $t_{1}<t_{2}$.

\section{Auxiliary Lemmas}

Suppose $u$ is a positive solution of (1.1), and as in [4], we introduce a function

$$
W=u^{-q}
$$

where $q$ is a positive constant to be determined later. For convenience, we always omit time variable $t$ and write $\mathcal{Q}_{t}$ for the partial derivative of $\mathcal{Q}$ relative to $t$. For example, throughout this paper, $\Delta, \nabla,|\cdot|$ mean the correspondence quantities with respect to $g(t)$. Write

$$
\square:=\Delta-\partial_{t} .
$$

A simple computation shows that

$$
\begin{aligned}
\nabla W & =-q u^{-q-1} \nabla u, \quad|\nabla W|^{2}=q^{2} u^{-2 q-2}|\nabla u|^{2} \\
W_{t} & =-q u^{-q-1} u_{t}, \quad \Delta W=q(q+1) u^{-q-2}|\nabla u|^{2}-q u^{-q-1} \Delta u .
\end{aligned}
$$

The relation (2.1) yields (see $[4,6]$ )

$$
|\nabla u|^{2}=\frac{|\nabla W|^{2}}{q^{2} W^{2+2 / q}}, \quad u_{t}=-\frac{W_{t}}{q W^{1+1 / q}},
$$

and hence

$$
\square W=\frac{q+1}{q} \frac{|\nabla W|^{2}}{W}+q h W^{1+\frac{1-p}{q}} .
$$

Since $|\nabla W|^{2} / W^{2}=q^{2}|\nabla u|^{2} / u^{2}$ and $h W^{(1-p) / q}=h u^{p-1}$, we consider again the same quantities as in $[4,6]$,

$$
\begin{aligned}
F_{0} & :=\frac{|\nabla W|^{2}}{W^{2}}+\alpha h W^{(1-p) / q}=|\nabla \ln W|^{2}+\alpha h W^{(1-p) / q}, \\
F_{1} & :=\frac{W_{t}}{W}=\partial_{t} \ln W \\
F & :=F_{0}+\beta F_{1} .
\end{aligned}
$$

Here $\alpha, \beta$ are two positive constants to be fixed later.

Introduce a 1-form $\underline{\boldsymbol{S}}_{g(t)}$ defined by

$$
\underline{S}_{g(t)}:=\operatorname{div}_{g(t)} S_{g(t)}-\frac{1}{2} \nabla_{g(t)}\left(\operatorname{tr}_{g(t)} S_{g(t)}\right) .
$$

Locally, one has

$$
\underline{\boldsymbol{S}}_{i}=\nabla^{j} S_{i j}-\frac{1}{2} \nabla_{i}\left(\operatorname{tr}_{g(t)} S_{g(t)}\right) .
$$


For example, if $S_{i j}=R_{i j}$, that is, (1.1) is the Ricci flow, we arrive at

$$
\underline{\boldsymbol{S}}_{i}=\nabla^{j} R_{i j}-\frac{1}{2} \nabla_{i} R_{g(t)}=\frac{1}{2} \nabla_{i} R_{g(t)}-\frac{1}{2} \nabla_{i} R_{g(t)}=0
$$

by the second contracted Bianchi identity. Thus, the quantity $\underline{\boldsymbol{S}}_{g(t)}$ measures whether $S_{i j}$ satisfies the second contracted Bianchi identity.

A analogous quantity like (2.7) also naturally appears in the general relativity, see, for example, Proposition 13.3 in [8].

Lemma 2.1. Suppose that $(M, g(t))_{t \in[0, T]}$ is a complete solution to the geometric flow (1.1) on $M$. If $u$ is a positive solution of (1.2), then

$$
\begin{aligned}
\square F_{1}= & \frac{2}{q}\left\langle\nabla F_{1}, \nabla \ln W\right\rangle+(1-p) h W^{\frac{1-p}{q}} \frac{W_{t}}{W}+q h_{t} W^{(1-p) / q} \\
& +2\left(1+\frac{1}{q}\right) S(\nabla \ln W, \nabla \ln W)-2\langle\underline{\boldsymbol{S}}, \nabla \ln W\rangle-\frac{2\left\langle S, \nabla^{2} W\right\rangle}{W} .
\end{aligned}
$$

Here div and $\operatorname{tr}$ are respectively divergence operator and trace operator of $g(t)$.

Proof. As in [6], we have

$$
\square F_{1}=\frac{\Delta W_{t}-W_{t t}}{W}-\frac{2\left\langle\nabla W, \nabla W_{t}\right\rangle}{W^{2}}-\frac{W_{t}\left(\Delta W-W_{t}\right)}{W^{2}}+\frac{2|\nabla W|^{2} W_{t}}{W^{3}} .
$$

Since $g(t)$ evolves under the geometric flow (1.1), it follows that

$$
\begin{aligned}
(\Delta W)_{t} & =\partial_{t}\left(g^{i j} \nabla_{i} \nabla_{j} W\right)=\left(\partial_{t} g^{i j}\right) \nabla_{i} \nabla_{j} W+g^{i j} \partial_{t}\left(\partial_{i} \partial_{j} W-\Gamma_{i j}^{k} \partial_{k} W\right) \\
& =2 S_{i j} \nabla^{i} \nabla^{j} W+\Delta\left(W_{t}\right)-g^{i j} \partial_{k} W \partial_{t} \Gamma_{i j}^{k} \\
& =\Delta\left(W_{t}\right)+2\left\langle S, \nabla^{2} W\right\rangle+2\langle\underline{S}, \nabla W\rangle
\end{aligned}
$$

using the fact that $g^{i j} \partial_{t} \Gamma_{i j}^{k}=-2 \nabla^{j} S_{j}{ }^{k}+\nabla^{k}(\operatorname{tr}(S))=-2 \underline{S}^{k}$. The term $\Delta W_{t}-$ $W_{t t}=\left(\Delta W-W_{t}\right)_{t}-2\left\langle S, \nabla^{2} W\right\rangle-2\langle\underline{\boldsymbol{S}}, \nabla W\rangle$ can be simplified as [6] into

$$
\begin{aligned}
\Delta W_{t}-W_{t t}= & 2\left(1+\frac{1}{q}\right) \frac{\left\langle\nabla W, \nabla W_{t}\right\rangle}{W}-\left(1+\frac{1}{q}\right) \frac{|\nabla W|^{2} W_{t}}{W^{2}}+q h_{t} W^{1+\frac{1-p}{q}} \\
& +\left(1+\frac{1}{q}\right) \frac{2 S(\nabla W, \nabla W)}{W}-2\langle\underline{\boldsymbol{S}}, \nabla W\rangle \\
& +h(q+1-p) W^{\frac{1-p}{q}} W_{t}-2\left\langle S, \nabla^{2} W\right\rangle .
\end{aligned}
$$

Plugging it into (2.9) yields

$$
\begin{aligned}
\square F_{1}= & \frac{2}{q} \frac{\left\langle\nabla W, \nabla W_{t}\right\rangle}{W^{2}}-\frac{2}{q} \frac{|\nabla W|^{2} W_{t}}{W^{3}}+(1-p) h W^{\frac{1-p}{q}-1} W_{t}+q h_{t} W^{\frac{1-p}{q}} \\
& +\left(1+\frac{1}{q}\right) \frac{2 S(\nabla W, \nabla W)}{W}-\frac{2\left\langle S, \nabla^{2} W\right\rangle}{W}-\frac{2\langle\underline{\boldsymbol{S}}, \nabla W\rangle}{W} .
\end{aligned}
$$

The desired equation (2.8) immediately follows.

Similarly, we can find the evolution equation of (2.5). 
Lemma 2.2. Suppose that $(M, g(t))_{t \in[0, T]}$ is a complete solution to the geometric flow (1.1) on $M$. If $u$ is a positive solution of (1.2), then

$$
\begin{aligned}
\square F_{0} \geq & 2(1-\epsilon)\left|\frac{\nabla^{2} W}{W}\right|^{2}+2\left(1-\frac{1}{\epsilon}\right)|\nabla \ln W|^{4}+\frac{2}{q}\left\langle\nabla F_{0}, \nabla \ln W\right\rangle \\
(2.10) \quad & -\frac{2 \alpha p}{q} W^{\frac{1-p}{q}}\langle\nabla \ln W, \nabla h\rangle+\alpha W^{\frac{1-p}{q}}\left(\Delta h-h_{t}\right)+\frac{2(\operatorname{Ric}-S)(\nabla W, \nabla W)}{W^{2}} \\
& +(1-p)\left(2-\frac{\alpha p}{q^{2}}\right) h W^{\frac{1-p}{q}}|\nabla \ln W|^{2}+\alpha(1-p) h^{2} W^{\frac{2(1-p)}{q}}
\end{aligned}
$$

where $\epsilon \in(0,1]$ is any given constant.

Proof. Recall from [6] that $\Delta F_{0}$ satisfies

$$
\begin{aligned}
\Delta F_{0}= & \frac{2\left|\nabla^{2} W\right|^{2}}{W^{2}}+\frac{2\langle\nabla W, \Delta \nabla W\rangle}{W^{2}}-8 \frac{\left\langle\nabla^{2} W, \nabla W \otimes \nabla W\right\rangle}{W^{3}}-\frac{2|\nabla W|^{2} \Delta W}{W^{3}} \\
(2.11)= & \frac{6|\nabla W|^{4}}{W^{4}}+\alpha W^{\frac{1-p}{q}} \Delta h+2 \alpha\left(\frac{1-p}{q}\right) W^{\frac{1-p}{q}-1}\langle\nabla W, \nabla h\rangle \\
& +\alpha\left(\frac{1-p}{q}\right)\left(\frac{1-p}{q}-1\right) h W^{\frac{1-p}{q}} \frac{|\nabla W|^{2}}{W^{2}}+\alpha\left(\frac{1-p}{q}\right) h W^{\frac{1-p}{q}-1} \Delta W .
\end{aligned}
$$

On the other hand, the time derivative of $F_{0}$ equals

$$
\begin{aligned}
\partial_{t} F_{0}= & \frac{2\left\langle\nabla W, \nabla W_{t}\right\rangle}{W^{2}}-\frac{2|\nabla W|^{2} W_{t}}{W^{3}}+\alpha h_{t} W^{\frac{1-p}{q}} \\
& +\alpha\left(\frac{1-p}{q}\right) h W^{\frac{1-p}{q}-1} W_{t}+\frac{2 S(\nabla W, \nabla W)}{W^{2}} .
\end{aligned}
$$

From (2.11), (2.12) and the Ricci identity $\Delta \nabla_{i} W=\nabla_{i} \Delta W+R_{i j} \nabla^{j} W$, we have

$$
\begin{aligned}
\square F_{0}= & \frac{2\left\langle\nabla W, \nabla\left(\Delta W-W_{t}\right)\right\rangle}{W^{2}}-\frac{\left.2|\nabla W|^{2}\left(\Delta W-W_{t}\right)\right\rangle}{W^{3}} \\
& +\left(\frac{2\left|\nabla^{2} W\right|^{2}}{W^{2}}-\frac{8\left\langle\nabla^{2} W, \nabla W \otimes \nabla W\right\rangle}{W^{3}}+\frac{6|\nabla W|^{4}}{W^{4}}\right) \\
& +\alpha W^{\frac{1-p}{q}}\left(\Delta h-h_{t}\right)+\alpha\left(\frac{1-p}{q}\right) h W^{\frac{1-p}{q}-1}\left(\Delta W-W_{t}\right) \\
& +2 \alpha\left(\frac{1-p}{q}\right) W^{\frac{1-p}{q}-1}\langle\nabla W, \nabla h\rangle+\frac{2(\operatorname{Ric}-S)(\nabla W, \nabla W)}{W^{2}} \\
& +\alpha\left(\frac{1-p}{q}\right)\left(\frac{1-p}{q}-1\right) h W^{\frac{1-p}{q}} \frac{\mid \nabla W^{2}}{W^{2}} .
\end{aligned}
$$

The following argument is the same as Lemma 2.2 in [6].

Combing Lemma 2.1 with Lemma 2.2, we get

Proposition 2.3. Suppose that $(M, g(t))_{t \in[0, T]}$ is a complete solution to the geometric flow (1.1) on $M$. If $u$ is a positive solution of (1.2), Define

$$
W=u^{-q}, \quad F=\frac{|\nabla W|^{2}}{W^{2}}+\alpha h W^{\frac{1-p}{q}}+\beta \frac{W_{t}}{W} .
$$


Then for all $\epsilon \in(0,1]$ we have

$$
\begin{aligned}
\square F \geq & 2(1-\epsilon)\left|\frac{\nabla^{2} W}{W}\right|^{2}+2\left(1-\frac{1}{\epsilon}\right)|\nabla \ln W|^{4}+\frac{2}{q}\langle\nabla F, \nabla \ln W\rangle \\
& +2 \beta\left(1+\frac{1}{q}\right) S(\nabla \ln W, \nabla \ln W)-\frac{2 \alpha p}{q} W^{\frac{1-p}{q}}\langle\nabla \ln W, \nabla h\rangle \\
& +(1-p)\left(2-\frac{\alpha p}{q^{2}}\right) h W^{\frac{1-p}{q}}|\nabla \ln W|^{2}+W^{\frac{1-p}{q}}\left[\alpha \Delta h+h_{t}(q \beta-\alpha)\right] \\
& +\alpha(1-p) h^{2} W^{\frac{2(1-p)}{q}}+\beta(1-p) h W^{\frac{1-p}{q}} \frac{W_{t}}{W}-2 \beta\left\langle S, \frac{\nabla^{2} W}{W}\right\rangle \\
& +2(\operatorname{Ric}-S)(\nabla \ln W, \nabla \ln W)-2 \beta\langle\underline{\boldsymbol{S}}, \nabla \ln W\rangle
\end{aligned}
$$

\section{Two SPECIAL CASES}

As in [6], we consider two special cases. The first special case of (2.14) is to choose

$$
\beta:=\frac{\alpha}{q}, \quad \alpha=\frac{k q^{2}}{p} .
$$

Then $q \beta-\alpha=0$ so that (2.14) becomes

$$
\begin{aligned}
\square F \geq & 2(1-\epsilon)\left|\frac{\nabla^{2} W}{W}\right|^{2}+2\left(1-\frac{1}{\epsilon}\right)|\nabla \ln W|^{4}+\frac{2 \alpha(1+q)}{q^{2}} S(\nabla \ln W, \nabla \ln W) \\
& +\frac{2}{q}\langle\nabla F, \nabla \ln W\rangle-\frac{2 \alpha p}{q} W^{\frac{1-p}{q}}\langle\nabla \ln W, \nabla h\rangle \\
(3.2) \quad & (1-p)\left(2-\frac{\alpha p}{q^{2}}\right) h W^{\frac{1-p}{q}}|\nabla \ln W|^{2}+\alpha W^{\frac{1-p}{q}} \Delta h \\
& +\alpha(1-p) h^{2} W^{\frac{2(1-p)}{q}}+\frac{\alpha(1-p)}{q} h W^{\frac{1-p}{q}} \frac{W_{t}}{W}-\frac{2 \alpha}{q}\left\langle S, \frac{\nabla^{2} W}{W}\right\rangle \\
& +2(\operatorname{Ric}-S)(\nabla \ln W, \nabla \ln W)-\frac{2 \alpha}{q}\langle\underline{\boldsymbol{S}}, \nabla \ln W\rangle .
\end{aligned}
$$

Recall the inequality in [6] (cf. (3.4))

$$
2\left|\frac{\nabla^{2} W}{W}\right|^{2}-\frac{2 \alpha}{q}\left\langle S, \frac{\nabla^{2} W}{W}\right\rangle \geq 2\left[\frac{a \alpha}{q}\left|\frac{\nabla^{2} W}{W}\right|^{2}-\frac{\alpha}{4 b q}|S|^{2}\right]
$$

for any positive real numbers $a, b$ satisfying $a+b=\frac{q}{\alpha}$, with the equality if $S=$ $2 b \nabla^{2} W / W$. Using the inequality $\left|\nabla^{2} W\right|^{2} \geq(\Delta W)^{2} / n$, we conclude from (3.2) and 
(3.3) that

$$
\begin{aligned}
\square F \geq & \frac{2}{n}\left(\frac{a \alpha}{q}-\epsilon\right)\left|\frac{\Delta W}{W}\right|^{2}+2\left(1-\frac{1}{\epsilon}\right)|\nabla \ln W|^{4}+\frac{2}{q}\langle\nabla F, \nabla \ln W\rangle \\
& +\frac{2 \alpha(1+q)}{q^{2}} S(\nabla \ln W, \nabla \ln W)-\frac{2 \alpha p}{q} W^{\frac{1-p}{q}}\langle\nabla \ln W, \nabla h\rangle \\
& +\alpha W^{\frac{1-p}{q}} \Delta h+(1-p)\left(2-\frac{\alpha p}{q^{2}}\right) h W^{\frac{1-p}{q}}|\nabla \ln W|^{2} \\
& +\alpha(1-p) h^{2} W^{\frac{2(1-p)}{q}}+\frac{\alpha(1-p)}{q} h W^{\frac{1-p}{q}} \frac{W_{t}}{W}-\frac{\alpha}{2 b q}|S|^{2} \\
& +2(\operatorname{Ric}-S)(\nabla \ln W, \nabla \ln W)-\frac{2 \alpha}{q}|\underline{S}||\nabla \ln W| .
\end{aligned}
$$

By (2.3), we get

$$
\frac{\Delta W}{W}=\frac{q+1}{q} \frac{|\nabla W|^{2}}{W^{2}}+\frac{W_{t}}{W}+q h W^{\frac{1-p}{q}}=\frac{q}{\alpha} F+\left(\frac{1+q}{q}-\frac{q}{\alpha}\right)|\nabla \ln W|^{2} .
$$

Because of the assumption $\alpha=k q^{2} / p$, we arrive at

$$
\frac{\Delta W}{W}=\frac{p}{k q} F+\left(\frac{1+q-p / k}{q}\right)|\nabla \ln W|^{2}
$$

Substituting (3.5) into (3.4), we obtain

Lemma 3.1. Suppose that $(M, g(t))_{t \in[0, T]}$ is a complete solution to the geometric flow (1.1) on $M$. If $u$ is a positive solution of (1.2), then

$$
\begin{aligned}
\square F \geq & \frac{2}{q}\langle\nabla F, \nabla \ln W\rangle+\frac{2}{n}\left(\frac{a k q}{p}-\epsilon\right) \frac{p^{2}}{k^{2} q^{2}} F^{2}+(1-p) h W^{\frac{1-p}{q}} F \\
& +\frac{4 p}{n}\left(\frac{a k q}{p}-\epsilon\right)\left(\frac{k+k q-p}{k^{2} q^{2}}\right) F|\nabla \ln W|^{2}-\frac{k q}{2 b p}|S|^{2} \\
& +2\left[\frac{1}{n}\left(\frac{a k q}{p}-\epsilon\right)\left(\frac{k+k q-p}{k q}\right)^{2}+\left(1-\frac{1}{\epsilon}\right)\right]|\nabla \ln W|^{4} \\
& +\frac{2 k(1+q)}{p} S(\nabla \ln W, \nabla \ln W)-2 q k W^{\frac{1-p}{q}}\langle\nabla \ln W, \nabla h\rangle \\
& +\frac{k q^{2}}{p} W^{\frac{1-p}{q}} \Delta h+(1-p)(1-k) h W^{\frac{1-p}{q}}|\nabla \ln W|^{2} \\
& +2(\operatorname{Ric}-S)(\nabla \ln W, \nabla \ln W)-\frac{2 k q}{p}|\underline{S}||\nabla \ln W|,
\end{aligned}
$$

where $\epsilon$ is a positive real number satisfying $\epsilon \in(0,1], p, q, k, a, b$ are positive real numbers such that $a+b=p / k q$, and

$$
W=u^{-q}, \quad F=\frac{|\nabla W|^{2}}{W^{2}}+\frac{k q^{2}}{p} h W^{\frac{1-p}{q}}+\frac{k q}{p} \frac{W_{t}}{W} .
$$

The second special case is to choose

$$
\beta:=\frac{2 \alpha}{q}, \quad \alpha=\frac{q^{2}}{p}
$$


in (2.14). Then the inequality (2.14) becomes

$$
\begin{aligned}
\square F \geq & 2(1-\epsilon)\left|\frac{\nabla^{2} W}{W}\right|^{2}+2\left(1-\frac{1}{\epsilon}\right)|\nabla \ln W|^{4}+\frac{2}{q}\langle\nabla F, \nabla \ln W\rangle \\
& +\frac{4(1+q)}{p} S(\nabla \ln W, \nabla \ln W)+(1-p) h W^{\frac{1-p}{q}}|\nabla \ln W|^{2} \\
& +\frac{q^{2}}{p} W^{\frac{1-p}{q}}\left(\Delta h+h_{t}\right)+q^{2}\left(\frac{1}{p}-1\right) h^{2} W^{\frac{2(1-p)}{q}}-2 q W^{\frac{1-p}{q}}\langle\nabla \ln W, \nabla h\rangle \\
& +2 q\left(\frac{1}{p}-1\right) h W^{\frac{1-p}{q}} \frac{W_{t}}{W}-\frac{4 q}{p}\left\langle S, \frac{\nabla^{2} W}{W}\right\rangle \\
& +2(\operatorname{Ric}-S)(\nabla \ln W, \nabla \ln W)-\frac{4 q}{p}\langle\underline{\boldsymbol{S}}, \nabla \ln W\rangle .
\end{aligned}
$$

For any positive real numbers $a, b$ with $a+b=q / 2 \alpha=p / 2 q$, we have (cf. [6])

$$
2\left|\frac{\nabla^{2} W}{W}\right|^{2}-\frac{4 q}{p}\left\langle S, \frac{\nabla^{2} W}{W}\right\rangle \geq \frac{4 a q}{p}\left|\frac{\nabla^{2} W}{W}\right|^{2}-\frac{q}{b p}|S|^{2} .
$$

Together (3.7), (3.8) with $\left|\nabla^{2} W\right|^{2} \geq(\Delta W)^{2} / n$ implies

$\square F \geq \frac{2}{n}\left(\frac{2 a q}{p}-\epsilon\right)\left|\frac{\Delta W}{W}\right|^{2}+2\left(1-\frac{1}{\epsilon}\right)|\nabla \ln W|^{4}+\frac{2}{q}\langle\nabla F, \nabla \ln W\rangle$

$$
\begin{aligned}
& +\frac{4(1+q)}{p} S(\nabla \ln W, \nabla \ln W)+(1-p) h W^{\frac{1-p}{q}}|\nabla \ln W|^{2}-\frac{q}{b p}|S|^{2} \\
& +\frac{q^{2}}{p} W^{\frac{1-p}{q}}\left(\Delta h+h_{t}\right)+q^{2}\left(\frac{1}{p}-1\right) h^{2} W^{\frac{2(1-p)}{q}}+2 q\left(\frac{1}{p}-1\right) h W^{\frac{1-p}{q}} \frac{W_{t}}{W} \\
& -2 q W^{\frac{1-p}{q}}\langle\nabla \ln W, \nabla h\rangle+2(\operatorname{Ric}-S)(\nabla \ln W, \nabla \ln W)-\frac{4 q}{p}\langle\underline{S}, \nabla \ln W\rangle .
\end{aligned}
$$

Substituting the identity (by (2.3))

$$
\frac{\Delta W}{W}=\frac{p}{2 q} F+\frac{q}{2} h W^{\frac{1-p}{q}}+\left(\frac{1+q-p / 2}{q}\right)|\nabla \ln W|^{2} .
$$

into (3.9) yields

$$
\begin{aligned}
\square F \geq & \frac{1}{2 n}\left(\frac{2 a q}{p}-\epsilon\right) \frac{p^{2}}{q^{2}} F^{2}+\frac{2 p}{n q^{2}}\left(\frac{2 a q}{p}-\epsilon\right)\left(1+q-\frac{p}{2}\right) F|\nabla \ln W|^{2} \\
& +\left[\frac{2}{n}\left(\frac{2 a q}{p}-\epsilon\right)\left(\frac{1+q-p / 2}{q}\right)^{2}+2\left(1-\frac{1}{\epsilon}\right)\right]|\nabla \ln W|^{4}-\frac{q}{b p}|S|^{2} \\
& +\frac{2}{q}\langle\nabla F, \nabla \ln W\rangle+\frac{4(1+q)}{p} S(\nabla \ln W, \nabla \ln W)+\frac{q^{2}}{p} W^{\frac{1-p}{q}}\left(\Delta h+h_{t}\right) \\
& +\frac{q^{2}}{2 n}\left(\frac{2 a q}{p}-\epsilon\right) h^{2} W^{\frac{2(1-p)}{q}}+\left[\frac{p}{n}\left(\frac{2 a q}{p}-\epsilon\right)+(1-p)\right] h W^{\frac{1-p}{q}} F \\
& +\frac{2}{n}\left(\frac{2 a q}{p}-\epsilon\right)\left(1+q-\frac{p}{2}\right) h W^{\frac{1-p}{q}}|\nabla \ln W|^{2}-2 q W^{\frac{1-p}{q}}\langle\nabla \ln W, \nabla h\rangle \\
& -\frac{4 q}{p}\langle\underline{\boldsymbol{S}}, \nabla \ln W\rangle+2(\operatorname{Ric}-S)(\nabla \ln W, \nabla \ln W) .
\end{aligned}
$$


The term $2 q W^{\frac{1-p}{q}}\langle\nabla \ln W, \nabla h\rangle$ is bounded from above by (where we assume that $h$ is nonnegative)

$$
\eta h W^{\frac{1-p}{q}}|\nabla \ln W|^{2}+\frac{q^{2}}{\eta} W^{\frac{1-p}{q}} \frac{|\nabla h|^{2}}{h}
$$

for any given $\eta>0$. Therefore

$$
\begin{aligned}
\square F \geq & \frac{1}{2 n}\left(\frac{2 a q}{p}-\epsilon\right) \frac{p^{2}}{q^{2}} F^{2}+\frac{2 p}{n q^{2}}\left(\frac{2 a q}{p}-\epsilon\right)\left(1+q-\frac{p}{2}\right) F|\nabla \ln W|^{2} \\
& +\left[\frac{2}{n}\left(\frac{2 a q}{p}-\epsilon\right)\left(\frac{1+q-p / 2}{q}\right)^{2}+2\left(1-\frac{1}{\epsilon}\right)\right]|\nabla \ln W|^{4}-\frac{q}{b p}|S|^{2} \\
& +\frac{2}{q}\langle\nabla F, \nabla \ln W\rangle+\frac{4(1+q)}{p} S(\nabla \ln W, \nabla \ln W) \\
& +\frac{q^{2}}{p} W^{\frac{1-p}{q}}\left(\Delta h+h_{t}-\frac{p}{\eta} \frac{|\nabla h|^{2}}{h}\right) \\
& +\frac{q^{2}}{2 n}\left(\frac{2 a q}{p}-\epsilon\right) h^{2} W^{\frac{2(1-p)}{q}}+\left[\frac{p}{n}\left(\frac{2 a q}{p}-\epsilon\right)+(1-p)\right] h W^{\frac{1-p}{q}} F \\
& +\left[\frac{2}{n}\left(\frac{2 a q}{p}-\epsilon\right)\left(1+q-\frac{p}{2}\right)-\eta\right] h W{ }^{\frac{1-p}{q}}|\nabla \ln W|^{2} \\
& +2(\operatorname{Ric}-S)(\nabla \ln W, \nabla \ln W)-\frac{4 q}{p}\langle\underline{\boldsymbol{S}}, \nabla \ln W\rangle .
\end{aligned}
$$

By choosing the same conditions on positive real numbers $p, q, a, b, \epsilon$ as in [6], Lemma 3.2, we obtain

Lemma 3.2. Suppose that $(M, g(t))_{t \in[0, T]}$ is a complete solution to the geometric flow (1.1) on an $n$-dimensional manifold $M$. Let $h(x, t)$ be a nonnegative function defined on $M \times[0, T]$ which is $C^{2}$ in $x$ and $C^{1}$ in $t$, and $\Delta_{g(t)} h+h_{t}-\frac{p}{\eta} \frac{\left|\nabla_{g(t)} h\right|_{g(t)}^{2}}{h} \geq 0$ on $M \times[0, T]$ for some $p, \eta>0$. Let $p, q, a, b, \epsilon$ be positive real numbers satisfying

(i) $q$ is a priori given positive real number;

(ii) $0<\epsilon \leq 1$;

(iii) $a+b=p / 2 q$;

(iv) either $0<\epsilon \leq \frac{2 a q-n(p-1)}{p}$ and $1<p<1+\frac{2 a q}{n}$ (then we choose $0<\eta \leq$ $\left.\frac{p-1}{2 p}\right)$, or $0<p \leq 1$ and $\frac{2 a q}{p}-\epsilon>0$ (then we choose $0<\eta \leq \frac{1}{n}\left(\frac{2 a q}{p}-\epsilon\right)$ ).

If $u$ is a positive solution of (1.2), $F\left(x_{0}, t_{0}\right)>0$ for some point $\left(x_{0}, t_{0}\right) \in M \times$ $[0, T]$, where

$$
F=\frac{|\nabla W|^{2}}{W^{2}}+\frac{q^{2}}{p} h W^{\frac{1-p}{q}}+\frac{2 q}{p} \frac{W_{t}}{W}
$$


then at the point $\left(x_{0}, t_{0}\right)$ we have

$$
\begin{aligned}
\square F \geq & \frac{1}{2 n}\left(\frac{2 a q}{p}-\epsilon\right) \frac{p^{2}}{q^{2}} F^{2}+\frac{2 p}{n q^{2}}\left(\frac{2 a q}{p}-\epsilon\right)\left(1+q-\frac{p}{2}\right) F|\nabla \ln W|^{2} \\
& +\left[\frac{2}{n}\left(\frac{2 a q}{p}-\epsilon\right)\left(\frac{1+q-p / 2}{q}\right)^{2}+2\left(1-\frac{1}{\epsilon}\right)\right]|\nabla \ln W|^{4}-\frac{q}{b p}|S|^{2} \\
& +\frac{2}{q}\langle\nabla F, \nabla \ln W\rangle+\frac{4(1+q)}{p} S(\nabla \ln W, \nabla \ln W) \\
& +2(\operatorname{Ric}-S)(\nabla \ln W, \nabla \ln W)-\frac{4 q}{p}\langle\underline{S}, \nabla \ln W\rangle .
\end{aligned}
$$

4. Gradient estimates And some Relative Results

In this section, we will use previous lemmas to get the gradient estimates for the positive solution of the equation (1.2) under the geometric flow.

Theorem 4.1. Suppose that $(M, g(t))_{t \in[0, T]}$ is a solution to the geometric flow (1.1) on $M$ with $-K_{1} g(t) \leq S_{g(t)} \leq K_{2} g(t),-K_{3} g(t) \leq \mathrm{Ric}_{g(t)}-S_{g(t)} \leq K_{3} g(t)$, and $\left|\underline{\boldsymbol{S}}_{g(t)}\right|_{g(t)} \leq K_{4}$ for some $K_{1}, K_{2}, K_{3}, K_{4}>0$ on $B_{2 R, T}$, with $\bar{K}:=\max \left\{K_{1}, K_{2}\right\}$. Let $h(x, t)$ be a function defined on $M \times[0, T]$ which is $C^{2}$ in $x$ and $C^{1}$ in $t$, satisfying $\Delta_{t} h \geq-\theta$ and $\left|\nabla_{t} h\right|_{t} \leq \gamma$ on $B_{2 R, T} \times[0, T]$ for some nonnegative constants $\theta$ and $\gamma$. If $u(x, t)$ is a positive smooth solution of (1.2) on $M \times[0, T]$, then

(i) for $0<p<1$, we have

$$
\begin{aligned}
\frac{\left|\nabla_{g(t)} u\right|_{g(t)}^{2}}{u^{2}}+\frac{h}{p} u^{p-1}-\frac{1}{p} \frac{u_{t}}{u} \leq & \frac{C_{1}}{p^{2} t}+\frac{n(1-p)}{p^{2}} M_{1} M_{2}+\frac{n\left[3 K_{1}+2\left(K_{3}+K_{4}\right) p\right]}{2 p^{2}(1-p)} \\
& +\frac{C_{1}}{p^{2}}\left(\frac{1}{R^{2}}+\frac{\sqrt{K_{1}+K_{3}}}{R}+\bar{K}+\frac{n}{p(1-p)}\right) \\
& +\left(\frac{n}{p}\right)^{3 / 2} \sqrt{\theta M_{2}}+\frac{\sqrt{n / K_{1}}}{p} \gamma M_{2}+\frac{n}{p^{2}} \sqrt{\frac{K_{4}}{2 n}}
\end{aligned}
$$

where $C_{1}$ is a positive constant depending only on $n$ and

$$
M_{1}:=\max _{B_{2 R, T}} h_{-}, \quad M_{2}:=\max _{B_{2 R, T}} u^{p-1}, \quad h_{-}:=\max (-h, 0) .
$$

(ii) for $p \geq 1$, we have

$$
\begin{aligned}
\frac{\left|\nabla_{g(t)} u\right|_{g(t)}^{2}}{u^{2}}+\frac{h}{p} u^{p-1}-\frac{1}{p} \frac{u_{t}}{t} \leq & \frac{k^{2} C_{2}}{p^{2} t}+\frac{n k^{2}(p-1)}{p^{2}} M_{4} M_{5}+\frac{k^{3} n}{k-p} M_{3} M_{4} \\
& +\frac{k^{2} C_{2}}{p^{2}}\left(\frac{1}{R^{2}}+\frac{\sqrt{K_{1}+K_{3}}}{R}+\bar{K}+\frac{k^{2} n}{p(k-p)}\right) \\
& +\frac{2 k^{3} n}{(k-2)}\left[K_{1}+\frac{p}{k}\left(K_{3}+K_{4}\right)\right]+\frac{k^{2} \sqrt{n} \gamma}{p} M_{4} \\
& +\left(\frac{k n}{p}\right)^{3 / 2} \sqrt{\theta M_{4}}+\frac{k^{2} n}{p^{2}}\left(\bar{K}+\sqrt{\frac{K_{4}}{2 n}}\right),
\end{aligned}
$$

where $k>p, C_{2}$ is a positive constant depending only on $n$ and

$$
M_{3}:=\max _{B_{2 R, T}} h_{-}, \quad M_{4}:=\max _{B_{2 R, T}} u^{p-1}, \quad M_{5}:=\max _{B_{2 R, T}} h .
$$


Proof. The proof is along the outline in $[1,4,5]$ and is identically as it in [6]. For completeness, we give a proof here. Firstly, we introduce a cut-off function (see $[3,1,5,6,7,10])$ on $B_{\rho, T}:=\left\{(x, t) \in M \times[0, T]: \operatorname{dist}_{g(t)}\left(x, x_{0}\right)<\rho\right\}$, where $\operatorname{dist}_{g(t)}\left(x, x_{0}\right)$ stands for the distance between $x$ and $x_{0}$ with respect to the metric $g(t)$, which satisfies a basic analytical result stated in the following lemma.

Lemma 4.2. Given $\tau \in(0, T]$, there exists a smooth function $\bar{\Psi}:[0, \infty) \times[0, T] \rightarrow$ $R$ satisfying the following requirements:

(1) The support of $\bar{\Psi}(r, t)$ is a subset of $[0, \rho] \times[0, T], 0 \leq \bar{\Psi}(r, t) \leq 1$ in $[0, \rho] \times[0, T]$, and $\bar{\Psi}(r, t)=1$ holds in $\left[0, \frac{\rho}{2}\right] \times[\tau, T]$.

(2) $\bar{\Psi}$ is decreasing as a radial function in the spatial variables.

(3) The estimate $\left|\partial_{t} \bar{\Psi}\right| \leq \frac{\bar{C}}{\tau} \bar{\Psi}^{1 / 2}$ is satisfied on $[0, \infty) \times[0, T]$ for some $\bar{C}>0$.

(4) The inequalities $-\frac{C_{\alpha}}{\rho} \bar{\Psi}^{\alpha} \leq \partial_{r} \bar{\Psi} \leq 0$ and $\left|\partial_{r}^{2} \bar{\Psi}\right| \leq \frac{C_{\alpha}}{\rho^{2}} \bar{\Psi}^{\alpha}$ hold on $[0, \infty) \times$ $[0, T]$ for every $\alpha \in(0,1)$ with some constant $C_{\alpha}$ dependent on $\alpha$.

Proof. See [1].

For the fixed $\tau \in(0, T]$, choose the above cut-off function $\bar{\Psi}$. Define $\Psi: M \times$ $[0, T] \rightarrow \mathbf{R}$ by setting

$$
\Psi(x, t):=\bar{\Psi}\left(\operatorname{dist}_{g(t)}\left(x, x_{0}\right), t\right)
$$

with $\rho:=2 R$ in Lemma 4.2. Consider the function $\varphi(x, t)=t F(x, t)$. Using the argument of Calabi [2], we may assume that the function $G(x, t):=\varphi(x, t) \Psi(x, t)$ with support in $B_{2 R, T}$ is smooth. Let $\left(x_{0}, t_{0}\right)$ be the point where $G$ achieves its maximum in the set $\left\{(x, t): 0 \leq t \leq \tau, d_{t}\left(x, x_{0}\right) \leq \rho\right\}$. Without loss of generality, assuming $G\left(x_{0}, t_{0}\right)>0$, we have

$$
\nabla G=0, \quad \partial_{t} G \geq 0, \quad \Delta G \leq 0
$$

at $\left(x_{0}, t_{0}\right)$. Now apply Lemma 4.2 and the Laplacian comparison theorem (observe that the hypothesis implies that $\left.-\left(K_{1}+K_{3}\right) g(t) \leq \operatorname{Ric}_{g(t)} \leq\left(K_{2}+K_{3}\right) g(t)\right)$, we have

$$
\begin{aligned}
\frac{|\nabla \Psi|^{2}}{\Psi} & \leq \frac{C_{1 / 2}^{2}}{\rho^{2}} \\
\Delta \Psi & \geq-\frac{C_{1 / 2} \Psi^{1 / 2}}{\rho^{2}}-\frac{C_{1 / 2} \Psi^{1 / 2}}{\rho}(n-1) \sqrt{K_{1}+K_{3}} \operatorname{coth}\left(\sqrt{K_{1}+K_{3}} \rho\right) \\
& \geq-\frac{d_{1}}{\rho^{2}}-\frac{d_{1} \Psi^{1 / 2}}{\rho} \sqrt{K_{1}+K_{3}}, \\
-\partial_{t} \Psi & \geq-\frac{\bar{C} \Psi^{1 / 2}}{\tau}-C_{1 / 2} \bar{K} \Psi^{1 / 2}
\end{aligned}
$$

where $C_{1 / 2}, \bar{C}$ and $d_{1}$ are positive constants depending only on $n$. It is easy to show that

$$
0 \geq \square G=\varphi \square \Psi+2\langle\nabla \varphi, \nabla \Psi\rangle+\Psi \square \varphi
$$


at $\left(x_{0}, t_{0}\right)$. Setting $p \in(0,1)$ and $k=1$ in Lemma 3.1, we obtain from $\square \varphi=$ $t \square F-\varphi / t$ that

$$
\begin{aligned}
& \square \varphi \geq \frac{2 p^{2}}{n t q^{2}}\left(\frac{a q}{p}-\epsilon\right) \varphi^{2}+\frac{4 p}{n}\left(\frac{a q}{p}-\epsilon\right)\left(\frac{1+q-p}{q^{2}}\right) \varphi|\nabla \ln W|^{2}-\frac{q^{2} \theta t}{p} W^{\frac{1-p}{q}} \\
&(4.4) \quad+2 t\left[\frac{1}{n}\left(\frac{a q}{p}-\epsilon\right)\left(\frac{1+q-p}{q}\right)^{2}+\left(1-\frac{1}{\epsilon}\right)\right]|\nabla \ln W|^{4}-\frac{n q \bar{K}^{2}}{2 b p} t \\
&-\frac{2(1+q) K_{1} t+2 p K_{3} t}{p}|\nabla \ln W|^{2}+\frac{2}{q}\langle\nabla \varphi, \nabla \ln W\rangle+(1-p) h W^{\frac{1-p}{q}} \varphi \\
&-2 q \gamma t W^{\frac{1-p}{q}}|\nabla \ln W|-\frac{2 q t}{p} K_{4}|\nabla \ln W|-\frac{\varphi}{t} .
\end{aligned}
$$

According to Hölder's inequality,

$$
\begin{aligned}
2 q \gamma t W^{\frac{1-p}{q}}|\nabla \ln W| & \leq \frac{(1+q) K_{1} t}{p}|\nabla \ln W|^{2}+\frac{p q^{2} \gamma^{2}}{(1+q) K_{1}} t W^{2 \frac{1-p}{q}} \\
\frac{2 q t}{p} K_{4}|\nabla \ln W| & \leq 2 K_{4} t|\nabla \ln W|^{2}+\frac{q^{2} K_{4}}{2 p^{2}} t
\end{aligned}
$$

we have

$$
\begin{aligned}
\square \varphi \geq & \frac{2 p^{2}}{n t q^{2}}\left(\frac{a q}{p}-\epsilon\right) \varphi^{2}+\frac{4 p}{n}\left(\frac{a q}{p}-\epsilon\right)\left(\frac{1+q-p}{q^{2}}\right) \varphi|\nabla \ln W|^{2}-\frac{q^{2} \theta t}{p} W^{\frac{1-p}{q}} \\
& +2 t\left[\frac{1}{n}\left(\frac{a q}{p}-\epsilon\right)\left(\frac{1+q-p}{q}\right)^{2}+\left(1-\frac{1}{\epsilon}\right)\right]|\nabla \ln W|^{4}-\frac{n q \bar{K}^{2}}{2 b p} t \\
& -\frac{3(1+q) K_{1} t+2 p\left(K_{3}+K_{4}\right) t}{p}|\nabla \ln W|^{2}+\frac{2}{q}\langle\nabla \varphi, \nabla \ln W\rangle-\frac{\varphi}{t} \\
& +(1-p) h W^{\frac{1-p}{q}} \varphi-\frac{p q^{2} \gamma^{2}}{(1+q) K_{1}} t W^{2 \frac{1-p}{q}}-\frac{q^{2} K_{4}}{2 p^{2}} t .
\end{aligned}
$$

Using Hölder's inequality again we have

$$
\begin{aligned}
& \frac{3(1+q) K_{1} t+2 p\left(K_{3}+K_{4}\right) t}{p}|\nabla \ln W|^{2} \\
\leq & \frac{n\left[3(1+q) K_{1}+2 p\left(K_{3}+K_{4}\right)\right]^{2} t}{8 p(a q-p \epsilon)}\left(\frac{q}{1-p}\right)^{2} \\
& +\frac{1}{n}\left(\frac{a q}{p}-\epsilon\right)\left(\frac{1-p}{q}\right)^{2} 2 t|\nabla \ln W|^{4} .
\end{aligned}
$$

Substituting (4.5) into (4.4) yields

$$
\begin{aligned}
\square \varphi \geq & \frac{2 p^{2}}{n t q^{2}}\left(\frac{a q}{p}-\epsilon\right) \varphi^{2}+\frac{4 p}{n}\left(\frac{a q}{p}-\epsilon\right)\left(\frac{1+q-p}{q^{2}}\right) \varphi|\nabla \ln W|^{2} \\
& +2 t\left[\frac{1}{n}\left(\frac{a q}{p}-\epsilon\right)\left(\frac{2-2 p+q}{q}\right)+\left(1-\frac{1}{\epsilon}\right)\right]|\nabla \ln W|^{4}-\frac{n q \bar{K}^{2}}{2 b p} t \\
& -\frac{n\left[3(1+q) K_{1}+2 p\left(K_{3}+K_{4}\right) p\right]^{2} t}{8 p(a q-p \epsilon)}\left(\frac{q}{1-p}\right)^{2}+\frac{2}{q}\langle\nabla \varphi, \nabla \ln W\rangle-\frac{\varphi}{t} \\
& +(1-p) h W^{\frac{1-p}{q}} \varphi-\frac{p q^{2} \gamma^{2}}{(1+q) K_{1}} t W^{2 \frac{1-p}{q}}-\frac{q^{2} \theta t}{p} W^{\frac{1-p}{q}}-\frac{q^{2} K_{4}}{2 p^{2}} t .
\end{aligned}
$$


Take $\epsilon \in(0,1 / 4)$ and choose $q$ so that $1 / q \geq n(1-\epsilon) / 2 \epsilon^{2}(1-p)$. For such a pair $(p, q)$, we may choose a positive real number $a$ such that $a q / p \geq 2 \epsilon$ and then the condition $a+b=p / q$ holds for some $b>0$ (because in this case $0<a q / p<1$ ). Under the above assumption, we have (as in [6])

$$
\frac{1}{n}\left(\frac{a q}{p}-\epsilon\right)\left(\frac{2-2 p+q}{q}\right)+\left(1-\frac{1}{\epsilon}\right) \geq 0 .
$$

and hence

$$
\begin{aligned}
\square \varphi \geq & \frac{2 p^{2}}{n t q^{2}}\left(\frac{a q}{p}-\epsilon\right) \varphi^{2}+\frac{4 p}{n}\left(\frac{a q}{p}-\epsilon\right)\left(\frac{1+q-p}{q^{2}}\right) \varphi|\nabla \ln W|^{2} \\
(4.6) \quad & -\frac{n q \bar{K}^{2}}{2 b p} t-\frac{n\left[3(1+q) K_{1}+2 p\left(K_{3}+K_{4}\right) p\right]^{2} t}{8 p(a q-p \epsilon)}\left(\frac{q}{1-p}\right)^{2}-\frac{\varphi}{t}-\frac{q^{2} K_{4}}{2 p^{2}} t \\
& -\frac{p q^{2} \gamma^{2}}{(1+q) K_{1}} t W^{2 \frac{1-p}{q}}-\frac{q^{2} \theta t}{p} W^{\frac{1-p}{q}}+\frac{2}{q}\langle\nabla \varphi, \nabla \ln W\rangle+(1-p) h W^{\frac{1-p}{q}} \varphi .
\end{aligned}
$$

Plugging (4.6) into (4.3) and using the estimate for $\square \Psi$, we arrive at, where $\rho:=2 R$,

$$
\begin{aligned}
0 \geq & \varphi \square \Psi-\frac{2 \varphi}{\Psi}|\nabla \Psi|^{2}+\Psi \square \varphi \\
\geq & \varphi d_{1}\left(-\frac{1}{\rho^{2}}-\frac{\sqrt{K_{1}+K_{3}}}{\rho}-\frac{1}{\tau}-\bar{K}\right)-\frac{2 d_{1}}{\rho^{2}} \varphi+\Psi \square \varphi \\
\geq & \frac{2 p^{2}}{n t q^{2}}\left(\frac{a q}{p}-\epsilon\right) \Psi \varphi^{2}+\frac{4 p}{n}\left(\frac{a q}{p}-\epsilon\right)\left(\frac{1+q-p}{q^{2}}\right) \varphi \Psi|\nabla \ln W|^{2} \\
& -\frac{n\left[3(1+q) K_{1}+2\left(K_{3}+K_{4}\right) p\right]^{2} t}{8 p(a q-p \epsilon)}\left(\frac{q}{1-p}\right)^{2}-\left(\frac{\theta M_{2}}{p}+\frac{p M_{2}^{2} \gamma^{2}}{(1+q) K_{1}}\right) q^{2} t \Psi \\
& -(1-p) M_{1} M_{2} \varphi \Psi-\frac{n q \bar{K}^{2}}{2 b p} t \Psi-\frac{\varphi \Psi}{t}+\varphi d_{2}\left(-\frac{1}{\rho^{2}}-\frac{\sqrt{K_{1}+K_{3}}}{\rho}-\frac{1}{\tau}-\bar{K}\right) \\
& -\frac{K_{4} q^{2}}{2 p^{2}} t \Psi-\frac{2}{q}\langle\nabla \Psi, \nabla \ln W\rangle \varphi,
\end{aligned}
$$

where $d_{1}, d_{2}$ are positive constants depending only on $n$, and

$$
M_{1}:=\sup _{B_{2 R, T}} h_{-}, \quad M_{2}:=\sup _{B_{2 R, T}} u^{p-1} .
$$

Multiplying the above inequality by $\Psi$ on both sides, we get, where $G=\varphi \Psi$

$$
\begin{aligned}
0 \geq & \frac{2 p^{2}}{n t q^{2}}\left(\frac{a q}{p}-\epsilon\right) G^{2}+\frac{4 p}{n}\left(\frac{a q}{p}-\epsilon\right)\left(\frac{1+q-p}{q^{2}}\right) G \Psi|\nabla \ln W|^{2} \\
(4.7) \quad & -\frac{n\left[3(1+q) K_{1}+2\left(K_{3}+K_{4}\right) p\right]^{2} t}{8 p(a q-p \epsilon)}\left(\frac{q}{1-p}\right)^{2}-\left(\frac{\theta M_{2}}{p}+\frac{p M_{2}^{2} \gamma^{2}}{(1+q) K_{1}}\right) t q^{2} \\
& -\frac{n q \overline{K^{2}}}{2 b p} t-\frac{G}{t}+G d_{2}\left(-\frac{1}{\rho^{2}}-\frac{\sqrt{K_{1}+K_{3}}}{\rho}-\frac{1}{\tau}-\bar{K}\right)-\frac{2}{q}\langle\nabla \Psi, \nabla \ln W\rangle G \\
& -(1-p) M_{1} M_{2} G-\frac{K_{4} q^{2}}{2 p^{2}} t .
\end{aligned}
$$


Using Hölder's inequality

$$
\begin{aligned}
\frac{2}{q}\langle\nabla \Psi, \nabla \ln W\rangle G \leq & \frac{4 p}{n}\left(\frac{a q}{p}-\epsilon\right)\left(\frac{1+q-p}{q^{2}}\right) G \Psi|\nabla \ln W|^{2} \\
& +\frac{\frac{1}{q^{2}}}{\frac{4 p}{n}\left(\frac{a q}{p}-\epsilon\right)\left(\frac{1+q-p}{q^{2}}\right)} \frac{|\nabla \Psi|^{2}}{\Psi} G
\end{aligned}
$$

the inequality (4.7) gives us the estimate (because $t \leq \tau$ )

$$
\begin{aligned}
0 \geq & \frac{2 p^{2}}{n q^{2}}\left(\frac{a q}{p}-\epsilon\right) G^{2}-(1-p) M_{1} M_{2} G t-d_{3} G \\
& -t\left[\frac{1}{\rho^{2}}+\frac{\sqrt{K_{1}+K_{3}}}{\rho}+\bar{K}+\frac{\frac{1}{q^{2}}}{\frac{4 p}{n}\left(\frac{a q}{p}-\epsilon\right)\left(\frac{1+q-p}{q^{2}}\right)}\right] d_{3} G \\
& -t^{2}\left[\frac{n\left[3(1+q) K_{1}+2\left(K_{3}+K_{4}\right) p\right]^{2}}{8 p(a q-p \epsilon)}\left(\frac{q}{1-p}\right)^{2}+\frac{q^{2}}{p} M_{2} \theta\right. \\
& \left.+\frac{q^{2} p}{(1+q) K_{1}}\left(M_{2} \gamma\right)^{2}+\frac{n q}{2 b p} \bar{K}^{2}+\frac{K_{4} q^{2}}{2 p^{2}}\right]
\end{aligned}
$$

for some positive constant $d_{3}$ depending only on $n$. The following inequality

$$
a G^{2}-b G-c \leq 0 \quad(a, b, c>0) \Longrightarrow G \leq \frac{b}{a}+\sqrt{\frac{c}{a}},
$$

implies

$$
\begin{aligned}
G \leq & \frac{d_{3}+(1-p) M_{1} M_{2} t+t d_{3}\left(\frac{1}{\rho^{2}}+\frac{\sqrt{K_{1}+K_{3}}}{\rho}+\bar{K}+\frac{\frac{1}{q^{2}}}{\frac{4 p}{n}\left(\frac{a q}{p}-\epsilon\right)\left(\frac{1+q-p}{q^{2}}\right)}\right)}{\frac{2 p^{2}}{n q^{2}}\left(\frac{a q}{p}-\epsilon\right)} \\
& +t \sqrt{\frac{\frac{n\left[3(1+q) K_{1}+2\left(K_{3}+K_{4}\right) p\right]^{2}}{8 p(a q-p \epsilon)}\left(\frac{q}{1-p}\right)^{2}+\frac{q^{2} M_{2} \theta}{p}+\frac{q^{2} p\left(M_{2} \gamma\right)^{2}}{(1+q) K_{1}}+\frac{n q \bar{K}^{2}}{2 b p}+\frac{K_{4} q^{2}}{2 p^{2}}}{\frac{2 p^{2}}{n q^{2}}\left(\frac{a q}{p}-\epsilon\right)}} .
\end{aligned}
$$

Recall the conditions on $p, q, \epsilon, a, b$ that

$$
0<p<1, \quad 0<\epsilon<\frac{1}{4}, \quad \frac{1}{q} \geq \frac{n(1-\epsilon)}{2 \epsilon^{2}(1-p)}, \quad a+b=\frac{p}{q}, \quad a \geq 2 \epsilon \frac{p}{q} .
$$

Choose $p, \epsilon, q$ as above and

$$
a=\left(\frac{1}{2}+2 \epsilon\right) \frac{p}{q}, \quad b=\left(\frac{1}{2}-2 \epsilon\right) \frac{p}{q} .
$$

The additional condition (4.9), plugging into the inequality for $G$, yields

$$
\begin{aligned}
G \leq & \frac{t n q^{2}\left[\frac{d_{3}}{t}+(1-p) M_{1} M_{2}+d_{3}\left(\frac{1}{\rho^{2}}+\frac{\sqrt{K_{1}+K_{3}}}{\rho}+\bar{K}+\frac{n}{2(1+2 \epsilon) p(1-p)}\right)\right]}{p^{2}(1+2 \epsilon)} \\
& +t \sqrt{\frac{n q^{4}}{p^{2}(1+2 \epsilon)}\left(\begin{array}{c}
\frac{n\left[3(1+q) K_{1}+2\left(K_{3}+K_{4}\right) p\right]^{2}}{4(1+2 \epsilon) p^{2}(1-p)^{2}}+\frac{M_{2} \theta}{p} \\
+\frac{p\left(M_{2} \gamma\right)^{2}}{(1+q) K_{1}}+\frac{n \bar{K}^{2}}{p^{2}(1-4 \epsilon)}+\frac{K_{4}}{2 p^{2}}
\end{array}\right)}
\end{aligned}
$$


at $\left(x_{0}, t_{0}\right)$. Since

$$
G=t q^{2}\left(\frac{|\nabla u|^{2}}{u^{2}}+\frac{h}{p} u^{p-1}-\frac{1}{p} \frac{u_{t}}{u}\right) \Psi
$$

and $q \leq 2 \epsilon^{2}(1-p) / n(1-\epsilon)$, it follows that, by letting $\epsilon \rightarrow 0$,

$$
\begin{aligned}
\frac{|\nabla u|^{2}}{u^{2}}+\frac{h}{p} u^{p-1}-\frac{1}{p} \frac{u_{t}}{u} \leq & \frac{d_{4}}{p^{2} t}+\frac{n(1-p)}{p^{2}} M_{1} M_{2} \\
& +\frac{d_{4}}{p^{2}}\left(\frac{1}{R^{2}}+\frac{\sqrt{K_{1}+K_{3}}}{R}+\bar{K}+\frac{n}{2 p(1-p)}\right) \\
& +\frac{n}{p^{2}} \sqrt{\frac{\frac{\left[3(1+q) K_{1}+2\left(K_{3}+K_{4}\right) p\right]^{2}}{4(1-p)^{2}}+\frac{p \theta}{n} M_{2}}{+\frac{(p \gamma)^{2}}{n K_{1}} M_{2}^{2}+\bar{K}^{2}+\frac{K_{4}}{2 n}}}
\end{aligned}
$$

on $B_{R, \tau}$, for some positive constant $d_{4}$ depending only on $n$. Because $\tau \in(0, T]$ was arbitrary, we arrive at

$$
\begin{aligned}
\frac{\left|\nabla_{t} u\right|_{t}^{2}}{u^{2}}+\frac{h}{p} u^{p-1}-\frac{1}{p} \frac{u_{t}}{u} \leq & \frac{d_{4}}{p^{2} t}+\frac{n(1-p)}{p^{2}} M_{1} M_{2}+\frac{n\left[3 K_{1}+2\left(K_{3}+K_{4}\right) p\right]}{2 p^{2}(1-p)} \\
& +\frac{d_{4}}{p^{2}}\left(\frac{1}{R^{2}}+\frac{\sqrt{K_{1}+K_{3}}}{R}+\bar{K}+\frac{n}{2 p(1-p)}\right) \\
& +\left(\frac{n}{p}\right)^{3 / 2} \sqrt{\theta M_{2}}+\frac{\sqrt{\frac{n}{K_{1}}}}{p} \gamma M_{2}+\frac{n}{p^{2}} \bar{K}+\frac{n}{p^{2}} \sqrt{\frac{K_{4}}{2 n}},
\end{aligned}
$$

on $B_{R, T}$. Arranging terms yields (4.1).

When $p \geq 1$, applying Lemma 3.1, we have

$$
\begin{aligned}
\square \varphi \geq & \frac{2 p^{2}}{n t k^{2} q^{2}}\left(\frac{a k q}{p}-\epsilon\right) \varphi^{2}+\frac{4 p}{n}\left(\frac{a k q}{p}-\epsilon\right)\left(\frac{k+k q-p}{k^{2} q^{2}}\right) \varphi|\nabla \ln W|^{2}-\frac{\varphi}{t} \\
& -\frac{k q^{2} \theta t}{p} W^{\frac{1-p}{q}}+2 t\left[\frac{1}{n}\left(\frac{a k q}{p}-\epsilon\right)\left(\frac{k+k q-p}{k q}\right)^{2}+\left(1-\frac{1}{\epsilon}\right)\right]|\nabla \ln W|^{4} \\
& -\frac{k n q \bar{K}^{2}}{2 b p} t+\frac{2}{q}\langle\nabla \varphi, \nabla \ln W\rangle+(1-p) h W^{\frac{1-p}{q}} \varphi \\
& -2 q k \gamma t W^{\frac{1-p}{q}}|\nabla \ln W|+(1-p)(1-k) t h W^{\frac{1-p}{q}}|\nabla \ln W|^{2} \\
& -\frac{2 k(1+q) K_{1} t+2 p\left(K_{3}+K_{4}\right) t}{p}|\nabla \ln W|^{2}-\frac{k^{2} q^{2} K_{4} t}{2 p^{2}},
\end{aligned}
$$

where $\epsilon \in(0,1]$ and $p, q, k, a, b$ are positive real numbers such that $a+b=p / k q$ and $k \geq 1$. Define

$$
M_{3}:=\max _{B_{2 R, T}} h_{-}, \quad M_{4}:=\max _{B_{2 R, T}} u^{p-1}, \quad M_{5}:=\max _{B_{2 R, T}} h,
$$

and

$$
\begin{aligned}
& M_{6}:=\quad \min _{q \geq 0} \min _{y \geq 0} \frac{1}{q^{2}}\left\{2\left[\frac{1}{n}\left(\frac{a k q}{p}-\epsilon\right)\left(\frac{k+k q-p}{k q}\right)^{2}+\left(1-\frac{1}{\epsilon}\right)\right] y^{2}\right. \\
&\left.-(p-1)(k-1) M_{3} M_{4} y-\frac{2 k(1+q) K_{1}+2 p\left(K_{3}+K_{4}\right)}{p} y-2 q k M_{4} \gamma y^{\frac{1}{2}}\right\} .
\end{aligned}
$$


Observe that $M_{6} \leq 0$. Therefore, we arrive at the following inequality

$$
\begin{aligned}
\square \varphi \geq & \frac{2 p^{2}}{n t k^{2} q^{2}}\left(\frac{a k q}{p}-\epsilon\right) \varphi^{2}+\frac{4 p}{n}\left(\frac{a k q}{p}-\epsilon\right)\left(\frac{k+k q-p}{k^{2} q^{2}}\right) \varphi|\nabla \ln W|^{2}-\frac{\varphi}{t} \\
& -\frac{k q^{2} \theta t}{p} M_{4}+\frac{2}{q}\langle\nabla \varphi, \nabla \ln W\rangle-(p-1) M_{4} M_{5} \varphi+M_{6} q^{2} t \\
& -\frac{k^{2} q^{2} K_{4}}{2 p^{2}} t-\frac{k n q \bar{K}^{2}}{2 b p} t .
\end{aligned}
$$

As before, using $0=\nabla G=\Psi \nabla \varphi+\varphi \nabla \Psi$ at $\left(x_{0}, t_{0}\right)$, we arrive at, where $\rho:=2 R$,

$$
\begin{aligned}
0 \geq & \varphi \square \Psi-2 \varphi \frac{|\nabla \Psi|^{2}}{\Psi}+\Psi \square \varphi \\
\geq & \varphi d_{1}\left(-\frac{1}{\rho^{2}}-\frac{\sqrt{K_{1}+K_{3}}}{\rho}-\frac{1}{\tau}-\bar{K}\right)-\frac{2 d_{1}}{\rho^{1}} \varphi+\Psi \square \varphi \\
\geq & \frac{2 p^{2}}{n t k^{2} q^{2}}\left(\frac{a k q}{p}-\epsilon\right) \Psi \varphi^{2}+\frac{4 p}{n}\left(\frac{a k q}{p}-\epsilon\right)\left(\frac{k+k q-p}{k^{2} q^{2}}\right) \varphi \Psi|\nabla \ln W|^{2} \\
& +M_{6} q^{2} \Psi t-\frac{2}{q}\langle\nabla \Psi, \nabla \ln W\rangle \varphi-\frac{k q^{2} \theta M_{4}}{p} \Psi t-(p-1) M_{4} M_{5} \Psi \varphi \\
& -\frac{\Psi \varphi}{t}+\varphi d_{2}\left(-\frac{1}{\rho^{2}}-\frac{\sqrt{K_{1}+K_{3}}}{\rho}-\frac{1}{t}-\bar{K}\right)-\left(\frac{k^{2} q^{2} K_{4}}{2 p^{2}}+\frac{k n q \bar{K}^{2}}{2 b p}\right) \Psi t
\end{aligned}
$$

for some positive constants $d_{1}, d_{2}$. Multiplying the above inequality by $\Psi$ on both sides, we get, where $G=\varphi \Psi$,

$$
\begin{aligned}
0 \geq & \frac{2 p^{2}}{n t k^{2} q^{2}}\left(\frac{a k q}{p}-\epsilon\right) G^{2}+\frac{4 p}{n}\left(\frac{a k q}{p}-\epsilon\right)\left(\frac{k+k q-p}{k^{2} q^{2}}\right) G \Psi|\nabla \ln W|^{2} \\
& +M_{6} q^{2} t-\frac{k q^{2} \theta t}{p} M_{4}-(p-1) M_{4} M_{5} G-\left(\frac{k^{2} q^{2} K_{4}}{2 p^{2}}+\frac{k n q \bar{K}^{2}}{2 b p}\right) t-\frac{G}{t} \\
& +G d_{2}\left(-\frac{1}{\rho^{2}}-\frac{\sqrt{K_{1}+K_{3}}}{\rho}-\frac{1}{\tau}-\bar{K}\right)-\frac{2}{q}\langle\nabla \Psi, \nabla \ln W\rangle G .
\end{aligned}
$$

Using Hölder's inequality, where we choose $a k q>\epsilon p$ and $k+k q>p$,

$$
\begin{aligned}
\frac{2}{q}\langle\nabla \Psi, \nabla \ln W\rangle G \leq & \frac{4 p}{n}\left(\frac{a k q}{p}-\epsilon\right)\left(\frac{k+k q-p}{k^{2} q^{2}}\right) G \Psi|\nabla \ln W|^{2} \\
& +\frac{\frac{1}{q^{2}}}{\frac{4 p}{n}\left(\frac{a k q}{p}-\epsilon\right)\left(\frac{k+k q-p}{k^{2} q^{2}}\right)} \frac{|\nabla \Psi|^{2}}{\Psi} G
\end{aligned}
$$

the inequality (4.10) gives the following estimate

$$
\begin{aligned}
0 \geq & \frac{2 p^{2}}{n k^{2} q^{2}}\left(\frac{a k q}{p}-\epsilon\right) G^{2}-(p-1) M_{4} M_{5} G t-d_{3} G \\
& -t\left[\frac{1}{\rho^{2}}+\frac{\sqrt{K_{1}+K_{3}}}{\rho}+\bar{K}+\frac{\frac{1}{q^{2}}}{\frac{4 p}{n}\left(\frac{a k q}{p}-\epsilon\right)\left(\frac{k+k q-p}{k^{2} q^{2}}\right)}\right] d_{3} G \\
& -t^{2}\left(\frac{k n q \bar{K}^{2}}{2 b p}+\frac{k^{2} q^{2} K_{4}}{2 p^{2}}+\frac{k q^{2}}{p} M_{4} \theta-M_{6} q^{2}\right)
\end{aligned}
$$


that is similar to $(4.8)$ at $\left(x_{0}, t_{0}\right)$, where $d_{3}$ is a positive constant. Hence

$$
\begin{aligned}
G \leq & \frac{d_{3}+(p-1) M_{4} M_{5} t+t d_{3}\left(\frac{1}{\rho^{2}}+\frac{\sqrt{K_{1}+K_{3}}}{\rho}+\bar{K}+\frac{\frac{1}{q^{2}}}{\frac{4 p}{n}\left(\frac{a k q}{p}-\epsilon\right)\left(\frac{k+k q-p}{k^{2} q^{2}}\right)}\right)}{\frac{2 p^{2}}{n k^{2} q^{2}}\left(\frac{a k q}{p}-\epsilon\right)} \\
& +t \sqrt{\frac{\frac{k n q \bar{K}^{2}}{2 b p}+\frac{k^{2} q^{2} K_{4}}{2 p^{2}}+\frac{k q^{2}}{p} M_{4} \theta-M_{6} q^{2}}{\frac{2 p^{2}}{n k^{2} q^{2}}\left(\frac{a k q}{p}-\epsilon\right)}}
\end{aligned}
$$

at $\left(x_{0}, t_{0}\right)$. Finally, we obtain

$$
\begin{aligned}
G \leq & \frac{t n k^{2} q^{2}}{p^{2}}\left[\frac{d_{3}}{t}+(p-1) M_{4} M_{5}+d_{3}\left(\frac{1}{\rho^{2}}+\frac{\sqrt{K_{1}+K_{3}}}{\rho}+\bar{K}+\frac{k^{2} n}{2 p(k+k q-p)}\right)\right] \\
& +t q^{2}\left[\frac{n k^{2}}{p^{2}}\left(\frac{k^{2} n \bar{K}^{2}}{p^{2}(1-2 \epsilon)}+\frac{k^{2} K_{4}}{2 p^{2}}+\frac{k}{p} M_{4} \theta-M_{6}\right)\right]^{1 / 2}
\end{aligned}
$$

by taking $a=\left(\epsilon+\frac{1}{2}\right) \frac{p}{k q}, b=\left(\frac{1}{2}-\epsilon\right) \frac{p}{k q}$ with $\epsilon \in(0,1 / 2)$ and $k \geq p$. As before, we conclude that

$$
\begin{aligned}
\frac{|\nabla u|^{2}}{u^{2}}+\frac{h}{p} u^{p-1}-\frac{1}{p} \frac{u_{t}}{t} \leq & \frac{k^{2} d_{4}}{p^{2} t}+\frac{n k^{2}(p-1)}{p^{2}} M_{4} M_{5} \\
& +\frac{k^{2} d_{4}}{p^{2}}\left(\frac{1}{R^{2}}+\frac{\sqrt{K_{1}+K_{3}}}{R}+\bar{K}+\frac{k^{2} n}{2 p(k-p)}\right) \\
& +\frac{k^{2} n}{p^{2}} \sqrt{-M_{6} \frac{p^{2}}{k^{2} n}+\frac{p \theta}{k n} M_{4}+\bar{K}^{2}+\frac{K_{4}}{2 n}}
\end{aligned}
$$

on $B_{R, \tau}$, for some positive constant $d_{4}$ depending only on $n$. Because $\tau \in(0, T]$ was arbitrary, we arrive at

$$
\begin{aligned}
\frac{|\nabla u|^{2}}{u^{2}}+\frac{h}{p} u^{p-1}-\frac{1}{p} \frac{u_{t}}{t} \leq & \frac{k^{2} d_{4}}{p^{2} t}+\frac{n k^{2}(p-1)}{p^{2}} M_{4} M_{5}+\frac{k^{2} n}{p^{2}}\left(\bar{K}+\sqrt{\frac{K_{4}}{2 n}}\right) \\
& +\frac{k^{2} d_{4}}{p^{2}}\left(\frac{1}{R^{2}}+\frac{\sqrt{K_{1}+K_{3}}}{R}+\bar{K}+\frac{k^{2} n}{2 p(k-p)}\right) \\
& +\frac{k \sqrt{n}}{p} \sqrt{-M_{6}}+\left(\frac{k n}{p}\right)^{3 / 2} \sqrt{\theta M_{4}}
\end{aligned}
$$

on $B_{R, \tau}$. In the following we shall show that $-M_{6}>0$ is bounded from above by some constant. For any $q, y \geq 0$ we have

$$
q^{2} M_{6} \geq\left[\frac{1}{n}\left(1+\frac{k-p}{k q}\right)^{2}+2\left(1-\frac{1}{\epsilon}\right)\right] y^{2}-A y-B y^{1 / 2}
$$

where

$$
A:=(p-1)(k-1) M_{3} M_{4}+\frac{2 k(1+q) K_{1}+2 p\left(K_{3}+K_{4}\right)}{p}, \quad B:=2 q k M_{4} \gamma
$$


Since $A y \leq \eta_{1} y^{2}+A^{2} / 4 \eta_{1}$ and $B y^{1 / 2} \leq \eta_{2} y+B^{2} / 4 \eta_{2}$ for any $\eta_{1}, \eta_{2}>0$, it follows that (as in [6]), where we choose $\eta_{1}=[(k-p) / k q]^{2} / 2 n$,

$$
\begin{aligned}
-M_{6} \leq & \frac{n k^{2}}{2(k-p)^{2}} \eta_{2}^{2}+\frac{n k^{2}}{2(k-p)^{2}}\left[(p-1)(k-1) M_{3} M_{4}\right. \\
& \left.+\frac{2 k(1+q) K_{1}+2 p\left(K_{3}+K_{4}\right)}{p}\right]^{2}+\frac{k^{2} M_{4}^{2} \gamma^{2}}{\eta_{2}}
\end{aligned}
$$

holds for any $q>0$. Because the right-hand side of (4.12) as a function of $q$ is increasing, letting $q \rightarrow 0$ yields

$$
\begin{aligned}
-M_{6} \leq & \frac{n k^{2}}{2(k-p)^{2}} \eta^{2}+\frac{k^{2} \gamma^{2} M_{4}^{2}}{\eta} \\
& +\frac{n k^{2}}{2(k-p)^{2}}\left[(p-1)(k-1) M_{3} M_{4}+\frac{2 k K_{1}}{p}+2\left(K_{3}+K_{4}\right)\right]^{2}
\end{aligned}
$$

where $\eta>0$. Using (4.13), we prove (4.2).

As an immediate consequence of the above theorem we have

Theorem 4.3. Suppose that $(M, g(t))_{t \in[0, T]}$ is a solution to the geometric flow (1.1) on $M$. Let $h(x, t)$ be a function defined on $M \times[0, T]$ which is $C^{2}$ in $x$ and $C^{1}$ in $t$.

(i) For $0<p<1$, assume that $h \geq 0$, $\left|\nabla_{t} h\right|_{t} \leq \gamma, \Delta_{t} h \geq 0$ along the geometric flow with $-K_{1} g(t) \leq S_{g(t)} \leq K_{2} g(t),-K_{3} g(t) \leq \mathrm{Ric}_{g(t)}-S_{g(t)} \leq$ $K_{3} g(t),\left|\underline{\boldsymbol{S}}_{g(t)}\right|_{g(t)} \leq K_{4}$ for some positive constants $\gamma, K_{1}, K_{2}, K_{3}, K_{4}$ with $\bar{K}:=$ $\max \left\{K_{1}, K_{2}\right\}$, along the geometric flow. If $u$ is a smooth positive function satisfying the nonlinear parabolic equation (1.2), then

$$
\begin{aligned}
\frac{\left|\nabla_{g(t)} u\right|_{g(t)}^{2}}{u^{2}}+\frac{h}{p} u^{p-1}-\frac{1}{p} \frac{u_{t}}{u} \leq & \frac{C_{1}}{p^{2} t}+\frac{C_{1}}{p^{3}(1-p)}+\frac{C_{1}}{p^{2}} \bar{K}+\frac{2 n K_{1}}{p^{2}(1-p)} \\
& +\frac{\sqrt{n / K_{1}}}{p} \gamma M+\frac{n}{p^{2}} \sqrt{\frac{K_{4}}{2 n}}+\frac{n\left(K_{3}+K_{4}\right)}{p(1-p)}
\end{aligned}
$$

for some positive constant $C_{1}$ depending only on $n$, where $M:=\max _{M \times[0, T]} u^{p-1}$.

(ii) For $p=1$, assume that $-K_{1} g(t) \leq S_{g(t)} \leq K_{2} g(t),-K_{3} g(t) \leq \mathrm{Ric}_{g(t)}-$ $S_{g(t)} \leq K_{3} g(t),\left|\underline{\boldsymbol{S}}_{g(t)}\right|_{g(t)} \leq K_{4}$ for some positive constants $K_{1}, K_{2}, \bar{K}_{3}, K_{4}$ with $\bar{K}:=\max \left\{K_{1}, K_{2}\right\}, h \geq 0, \Delta_{g(t)} h \geq-\theta$ ( $\theta$ is nonnegative $)$, and $\left|\nabla_{g(t)} h\right|_{g(t)} \leq \gamma$ ( $\gamma$ is nonnegative), along the geometric flow. If $u$ is a smooth positive function satisfying the nonlinear parabolic equation (1.2), then

$$
\frac{\left|\nabla_{g(t)} u\right|_{g(t)}^{2}}{u^{2}}+h-\frac{u_{t}}{u} \leq \frac{C_{2}}{t}+C_{2}\left(1+K_{1}+K_{2}+K_{3}+K_{4}+\bar{K}+\gamma+\sqrt{\theta}\right)
$$

for some positive constant $C_{2}$ depending only on $n$.

(iii) For $p>1$, assume that $-K_{1} g(t) \leq S_{g(t)} \leq K_{2} g(t),-K_{3} g(t) \leq \mathrm{Ric}_{g(t)}-$ $S_{g(t)} \leq K_{3} g(t),\left|\underline{\boldsymbol{S}}_{g(t)}\right|_{g(t)} \leq K_{4}$ for some positive constants $\gamma, K_{1}, K_{2}, K_{3}, K_{4}$ with $\bar{K}:=\max \left\{K_{1}, K_{2}\right\} . \Delta_{g(t)} h \geq-\theta,\left|\nabla_{g(t)} h\right|_{g(t)} \leq \gamma$, and $-k_{1} \leq h \leq k_{2}$, where $\theta, \gamma, k_{1}, k_{2}>0$, along the geometric flow. If $u$ is a bounded smooth positive function 
satisfying the nonlinear parabolic equation (1.2), then

$$
\begin{aligned}
\frac{\left|\nabla_{g(t)} u\right|_{g(t)}^{2}}{u^{2}}+\frac{h}{p} u^{p-1}-\frac{1}{p} \frac{u_{t}}{u} \leq & \left(\frac{k}{p}\right)^{2} \frac{C_{3}}{t}+\left(\frac{k}{p}\right)^{3} \frac{k}{k-p} C_{3}+\left(\frac{k}{p}\right)^{2} C_{3}(\bar{K}+ \\
& \left.+\frac{k}{k-p}\left(K_{1}+K_{3}+K_{4}\right)+\sqrt{\frac{K_{4}}{2 n}}\right) \\
& +\left(\frac{k}{p}\right)^{2} n(p-1) k_{2} M+\frac{k^{3} n}{k-p} k_{1} M \\
& +\frac{k^{2} \sqrt{n}}{p} \gamma M+\left(\frac{k n}{p}\right)^{3 / 2} \sqrt{\theta M},
\end{aligned}
$$

for some positive constant $C_{3}$ depending only on $n$, where $M:=\max _{M \times[0, T]} u^{p-1}$ and $k>p$. In particular, taking $k=2 p$, we get

$$
\begin{aligned}
\frac{\left|\nabla_{g(t)} u\right|_{g(t)}^{2}}{u^{2}}+\frac{h}{p} u^{p-1}-\frac{1}{p} \frac{u_{t}}{u} \leq & \frac{C_{4}}{t}+C_{5}\left(1+K_{1}+K_{2}+K_{3}+K_{4}+\bar{K}\right) \\
& +C_{4} p^{2}\left[\left(k_{1}+k_{2}\right) M+\gamma M+\sqrt{\theta M}\right],
\end{aligned}
$$

for some positive constant $C_{4}$ depending only on $n$.

In Lemma 3.2, we required that

$$
\Delta_{g(t)} h+h_{t}-\frac{p}{\eta} \frac{\left|\nabla_{g(t)} h\right|_{g(t)}^{2}}{h} \geq 0
$$

for some positive constant $p, \eta$. In the following proof, we shall see that when $0<p \leq \frac{2 n}{2 n-1}$, we need only to assume that

$$
\Delta_{g(t)}+h_{t}-2 C_{n, p} p \frac{\left|\nabla_{g(t)} h\right|_{g(t)}^{2}}{h} \geq 0
$$

where

$$
C_{n, p}=\left\{\begin{array}{cl}
n, & p \leq 1 \\
\frac{p}{p-1}, & p>1
\end{array}\right.
$$

Theorem 4.4. Suppose that $(M, g(t))_{t \in[0, T]}$ is a solution to the geometric flow (1.1) on $M$, satisfying $-K_{1} g(t) \leq S_{g(t)} \leq K_{2} g(t),-K_{3} g(t) \leq \mathrm{Ric}_{g(t)}-S_{g(t)} \leq K_{3} g(t)$, $\left|\nabla_{t} \underline{\boldsymbol{S}}_{g(t)}\right|_{g(t)} \leq K_{4}$, for some $K_{1}, K_{2}, K_{3}, K_{4}>0$, with $\bar{K}:=\max \left\{K_{1}, K_{2}\right\}$. Let $h(x, t)$ be a nonnegative function defined on $M \times[0, T]$ which is $C^{2}$ in $x$ and $C^{1}$ in $t, \Delta_{g(t)} h+h_{t}-2 C_{n, p} p \frac{\left|\nabla_{g(t)} h\right|_{g(t)}^{2}}{h} \geq 0$ on $M \times[0, T]$ (where $C_{n, p}=\frac{p}{p-1}$ if $p>1$ and $C_{n, p}=n$ if $\left.p \leq 1\right)$, and $0<p \leq \frac{2 n}{2 n-1}(n \geq 3)$. If $u$ is a positive solution of (1.2), then

$$
\begin{aligned}
\frac{\left|\nabla_{g(t)} u\right|_{g(t)}^{2}+\frac{h}{p} u^{p-1}-\frac{2}{p} \frac{u_{t}}{u} \leq}{u^{2}} & \frac{C}{p^{2} t}+\frac{8 n}{p^{2}} \bar{K}+\frac{8 n}{p^{2}} \sqrt{\frac{2 n}{p(2-p)} K_{1}} \\
& +\frac{4 n}{p(2-p)}\left(K_{1}+K_{3}+K_{4}\right)+\frac{1}{p^{2}} \sqrt{8 n K_{4}}
\end{aligned}
$$

for some positive constant $C$ depending only on $n$. 
Proof. As in the proof of Theorem 4.1, we have

$$
\begin{aligned}
\square \varphi \geq & \frac{1}{2 n t}\left(\frac{2 a q}{p}-\epsilon\right) \frac{p^{2}}{q^{2}} \varphi^{2}+\frac{2 p}{n q^{2}}\left(\frac{2 a q}{p}-\epsilon\right)\left(1+q-\frac{p}{2}\right) \varphi|\nabla \ln W|^{2} \\
& +2 t\left[\frac{1}{n}\left(\frac{2 a q}{p}-\epsilon\right)\left(\frac{1+q-p / 2}{q}\right)^{2}+\left(1-\frac{1}{\epsilon}\right)\right]|\nabla \ln W|^{4}-\frac{q n t}{b p} \bar{K}^{2}-\frac{\varphi}{t} \\
& +\frac{2}{q}\langle\nabla \varphi, \nabla \ln W\rangle-\frac{4(1+q) K_{1}+2\left(K_{3}+K_{4}\right) p}{p} t|\nabla \ln W|^{2}-\frac{2 q^{2} K_{4} t}{p^{2}}
\end{aligned}
$$

where $\varphi=t F$, from Lemma 3.2. Using Hölder's inequality

$$
\begin{aligned}
\frac{4(1+q) K_{2}+2 K_{1}}{p} t|\nabla \ln W|^{2} \leq & \frac{1}{n}\left(\frac{2 a q}{p}-\epsilon\right)\left(\frac{1-p / 2}{q}\right)^{2} 2 t|\nabla \ln W|^{4} \\
& +\frac{2 n t\left[(1+q) K_{1}+\frac{1}{2}\left(K_{3}+K_{4}\right) p\right]^{2}}{p(2 a q-p \epsilon)}\left(\frac{q}{1-p / 2}\right)^{2},
\end{aligned}
$$

we see that

$$
\begin{aligned}
\square \varphi \geq & \frac{1}{2 n t}\left(\frac{2 a q}{p}-\epsilon\right) \frac{p^{2}}{q^{2}} \varphi^{2}+\frac{2 p}{n q^{2}}\left(\frac{2 a q}{p}-\epsilon\right)\left(1+q-\frac{p}{2}\right) \varphi|\nabla \ln W|^{2} \\
& -\frac{n q \bar{K}^{2} t}{b p}-\frac{2 n t\left[(1+q) K_{1}+\frac{1}{2}\left(K_{3}+K_{4}\right) p\right]^{2}}{p(2 a q-p \epsilon)}\left(\frac{q}{1-p / 2}\right)^{2} \\
& +\frac{2}{q}\langle\nabla \varphi, \nabla \ln W\rangle-\frac{2 q^{2} K_{4} t}{p^{2}}-\frac{\varphi}{t} .
\end{aligned}
$$

Writing $G:=\varphi \Psi$ and using $\square G=\varphi \square \Psi-2 \varphi|\nabla \Psi|^{2} / \Psi+\Psi \square \varphi$, as before, we arrive at

$$
\begin{aligned}
0 \geq & \frac{p^{2}}{2 n t q^{2}}\left(\frac{2 a q}{p}-\epsilon\right) G^{2}+\frac{2 p}{n}\left(\frac{2 a q}{p}-\epsilon\right)\left(\frac{1+q-p / 2}{q^{2}}\right) G \Psi|\nabla \ln W|^{2} \\
& -\frac{2 n t\left[(1+q) K_{1}+\frac{1}{2}\left(K_{3}+K_{4}\right) p\right]^{2}}{p(2 a q-p \epsilon)}\left(\frac{q}{1-p / 2}\right)^{2}-\frac{n q \bar{K}^{2}}{b p} t-\frac{G}{t} \\
& -\frac{2}{q}\langle\nabla \Psi, \nabla \ln W\rangle G+G d_{1}\left(-\frac{1}{\rho^{2}}-\frac{\sqrt{K_{1}+K_{3}}}{\rho}-\frac{1}{\tau}-\bar{K}\right)-\frac{2 q^{2} K_{4}}{p^{2}} t,
\end{aligned}
$$

for some positive constant $d_{1}$ depending only on $n$. Plugging the inequality

$$
\begin{aligned}
\frac{2}{q}\langle\nabla \Psi, \nabla \ln W\rangle G \leq & \frac{2 p}{n}\left(\frac{2 a q}{p}-\epsilon\right)\left(\frac{1+q-p / 2}{q^{2}}\right) G \Psi|\nabla \ln W|^{2} \\
& +\frac{\frac{1}{q^{2}}}{\frac{2 p}{n}\left(\frac{2 a q}{p}-\epsilon\right)\left(\frac{1+q-p / 2}{q^{2}}\right)} \frac{|\nabla \Psi|^{2}}{\Psi} G
\end{aligned}
$$

into (4.19) yields

$$
\begin{aligned}
0 \geq & \frac{p^{2}}{2 n q^{2}}\left(\frac{2 a q}{p}-\epsilon\right) G^{2}-d_{2} G \\
& -t\left[\frac{1}{\rho^{2}}+\frac{\sqrt{K_{1}+K_{3}}}{\rho}+\bar{K}+\frac{n}{2(2 a q-p \epsilon)(1+q-p / 2)}\right] d_{2} G \\
& -t^{2}\left[\frac{2 n\left[(1+q) K_{1}+\frac{1}{2}\left(K_{3}+K_{4}\right) p\right]^{2}}{p(2 a q-p \epsilon)}\left(\frac{q}{1-p / 2}\right)^{2}+\frac{n q}{b p} \bar{K}^{2}+\frac{2 q^{2} K_{4}}{p^{2}}\right]
\end{aligned}
$$


for some positive constant $d_{2}$ depending only on $n$. Hence

$$
\begin{aligned}
G \leq & \frac{d_{2}+t\left(\frac{1}{\rho^{2}}+\frac{\sqrt{K_{1}+K_{3}}}{\rho}+\bar{K}+\frac{n}{2(2 a q-p \epsilon)(1+q-p / 2)}\right)}{\frac{p^{2}}{2 n q^{2}}\left(\frac{2 a q}{p}-\epsilon\right)} \\
& +t \sqrt{\frac{\frac{2 n\left[(1+q) K_{1}+\frac{1}{2}\left(K_{3}+K_{4}\right) p\right]^{2}}{p(2 a q-p \epsilon)}\left(\frac{q}{1-p / 2}\right)^{2}+\frac{n q}{b p} \bar{K}^{2}+\frac{2 q^{2} K_{4}}{p^{2}}}{\frac{p^{2}}{2 n q^{2}}\left(\frac{2 a q}{p}-\epsilon\right)}}
\end{aligned}
$$

The above calculation is based on the assumption that

$$
\Delta_{t} h+h_{t}-\frac{p}{\eta} \frac{\left|\nabla_{t} h\right|_{t}^{2}}{h} \geq 0
$$

for some positive constant $\eta, p>0$. We now choose appropriate constants, together with the our assumption that

$$
\Delta_{t} h+h_{t}-2 C_{n, p} p \frac{\left|\nabla_{t} h\right|_{t}^{2}}{h} \geq 0
$$

to verify this assumption in Lemma 3.2. Recall the conditions on $p, q, \epsilon, a, b$. First we consider the case,

$$
q>0, \quad 0<\epsilon \leq 1, \quad a+b=\frac{p}{2 q}, \quad 0<p \leq 1, \quad 0<\epsilon<\frac{2 a q}{p} .
$$

Choose

$$
a=\left(\epsilon+\frac{1}{2}\right) \frac{p}{2 q}, \quad b=\left(\frac{1}{2}-\epsilon\right) \frac{p}{2 q}, \quad 0<\epsilon<\frac{1}{2} .
$$

Then we can choose $\eta=\frac{1}{n}\left(\frac{2 a q}{p}-\epsilon\right)=\frac{1}{2 n}$ so that $p / \eta=2 n p>2 p$, and furthermore

$$
\begin{aligned}
G \leq & \frac{4 n q^{2} t}{p^{2}}\left[\frac{d_{2}}{t}+\left(\frac{1}{\rho^{2}}+\frac{\sqrt{K_{1}+K_{3}}}{\rho}+\bar{K}+\frac{n}{p\left(1+q-\frac{p}{2}\right)}\right)\right] \\
& +\frac{4 n q^{2} t}{p^{2}} \sqrt{\frac{1}{1-2 \epsilon} \bar{K}^{2}+\frac{\left[(1+q) K_{1}+\frac{1}{2}\left(K_{3}+K_{4}\right) p\right]^{2}}{\left(1-\frac{p}{2}\right)^{2}}+\frac{K_{4}}{2 n}} .
\end{aligned}
$$

Letting $\epsilon \rightarrow 0$ and $R \rightarrow \infty$ implies

$$
\begin{aligned}
\frac{p^{2}}{4 n}\left(\frac{|\nabla u|^{2}}{u^{2}}+\frac{h}{p} u^{p-1}-\frac{2}{p} \frac{u_{t}}{t}\right) \leq & \frac{d_{2}}{t}+\bar{K}+\frac{n}{p\left(1+q-\frac{p}{2}\right)} \\
& +\sqrt{\bar{K}^{2}+\frac{\left[(1+q) K_{1}+\frac{1}{2}\left(K_{3}+K_{4}\right) p\right]^{2}}{\left(1-\frac{p}{2}\right)^{2}}+\frac{K_{4}}{2 n}}
\end{aligned}
$$

Now we minimize the above inequality for any $q>0$ by the following observation

$$
\begin{aligned}
\frac{p^{2}}{4 n}\left(\frac{|\nabla u|^{2}}{u^{2}}+\frac{h}{p} u^{p-1}-\frac{2}{p} \frac{u_{t}}{t}\right) \leq & \frac{d_{2}}{t}+2 \bar{K}+\frac{n}{p\left(1+q-\frac{p}{2}\right)}+\frac{1+q-\frac{p}{2}}{1-\frac{p}{2}} K_{1} \\
& +\frac{p}{2-p}\left(K_{1}+K_{3}+K_{4}\right)+\sqrt{\frac{K_{4}}{2 n}} .
\end{aligned}
$$


Hence

$$
\begin{aligned}
\frac{p^{2}}{4 n}\left(\frac{|\nabla u|^{2}}{u^{2}}+\frac{h}{p} u^{p-1}-\frac{2}{p} \frac{u_{t}}{t}\right) \leq & \frac{d_{2}}{t}+2 \bar{K}+2 \sqrt{\frac{n}{p\left(1-\frac{p}{2}\right)} K_{1}} \\
& +\frac{p}{2-p}\left(K_{1}+K_{3}+K_{4}\right)+\sqrt{\frac{K_{4}}{2 n}} \\
= & \frac{d_{2}}{t}+2 \bar{K}+2 \sqrt{\frac{2 n}{p(2-p)} K_{1}} \\
& +\frac{p}{2-p}\left(K_{1}+K_{3}+K_{4}\right)+\sqrt{\frac{K_{4}}{2 n}}
\end{aligned}
$$

Next we consider the second case; that is,

(4.22) $q>0, \quad 0<\epsilon \leq 1, \quad a+b=\frac{p}{2 q}, \quad 1<p<1+\frac{2 a q}{n}, \quad 0<\epsilon \leq \frac{2 a q-n(p-1)}{p}$.

We have proved that $1<p<\frac{n}{n-1} \leq 2$ and $1+q-\frac{p}{2}>0$ in this case. Choose

$$
a=\left(\epsilon+\frac{1}{2}\right) \frac{p}{2 q}, \quad b=\left(\frac{1}{2}-\epsilon\right) \frac{p}{2 q}, \quad 0<\epsilon<\frac{1}{2}, \quad 1<p \leq \frac{2 n}{2 n-1}
$$

and $\eta=\frac{p-1}{2 p} \in\left(0, \frac{1}{4 n}\right]$ so that $p / \eta=2 p \frac{p}{p-1}>2 p$. This choice of positive constants $a, b, p, q, \epsilon$ satisfies the mentioned condition (4.22). Then we obtain the same inequality

$$
\begin{aligned}
G \leq & \frac{4 n q^{2} t}{p^{2}}\left[\frac{d_{2}}{t}+\left(\frac{1}{\rho^{2}}+\frac{\sqrt{K_{1}+K_{3}}}{\rho}+\bar{K}+\frac{n}{p\left(1+q-\frac{p}{2}\right)}\right)\right] \\
& +\frac{4 n q^{2} t}{p^{2}} \sqrt{\frac{1}{1-2 \epsilon} \bar{K}^{2}+\frac{\left[(1+q) K_{1}+\frac{1}{2}\left(K_{3}+K_{4}\right) p\right]^{2}}{\left(1-\frac{p}{2}\right)^{2}}+\frac{K_{4}}{2 n}} .
\end{aligned}
$$

Letting $\epsilon \rightarrow 0$ and $R \rightarrow \infty$, and minimizing over all $q>0$, we obtain

$$
\begin{aligned}
\frac{p^{2}}{4 n}\left(\frac{|\nabla u|^{2}}{u^{2}}+\frac{h}{p} u^{p-1}-\frac{2}{p} \frac{u_{t}}{t}\right) \leq & \frac{d_{2}}{t}+2 \bar{K}+2 \sqrt{\frac{2 n}{p(2-p)} K_{1}} \\
& +\frac{p}{2-p}\left(K_{1}+K_{3}+K_{4}\right)+\sqrt{\frac{K_{4}}{2 n}}
\end{aligned}
$$

In both cases, we proved Theorem 4.4.

Corollary 4.5. Suppose that $(M, g(t))_{t \in[0, T]}$ is a solution to the geometric flow (1.1) on $M$, satisfying $0 \leq S_{g(t)} \leq K_{2} g(t),-K_{3} \leq \mathrm{Ric}_{g(t)}-S_{g(t)} \leq K_{3} g(t)$, $\left|\nabla_{g(t)} \underline{\boldsymbol{S}}_{g(t)}\right|_{g(t)} \leq K_{4}$, for some positive constants $K_{2}, K_{3}, K_{4}$. Let $h(x, t)$ be a nonnegative function defined on $M \times[0, T]$ which is $C^{2}$ in $x$ and $C^{1}$ in $t, \Delta_{g(t)} h+$ $h_{t}-2 C_{n, p} p \frac{\left|\nabla_{g(t)} h\right|_{g(t)}^{2}}{h} \geq 0$ on $M \times[0, T]$ (where $C_{n, p}=\frac{p}{p-1}$ if $p>1$ and $C_{n, p}=n$ if $p \leq 1)$, and $0<p \leq \frac{2 n}{2 n-1}(n \geq 3)$. If $u$ is a positive solution of (1.2), then

$$
\frac{\left|\nabla_{g(t)} u\right|_{g(t)}^{2}}{u^{2}}+\frac{h}{p} u^{p-1}-\frac{2}{p} \frac{u_{t}}{t} \leq \frac{C}{p^{2} t}+\frac{8 n}{p^{2}} K_{2}+\frac{4 n}{p(2-p)}\left(K_{3}+K_{4}\right)+\frac{1}{p^{2}} \sqrt{8 n K_{4}}
$$

for some positive constant $C$ depending only on $n$. 
Under the hypotheses of Theorem 4.4 , we let $f:=\ln u$. Then

$$
\begin{aligned}
\left|\nabla_{t} f\right|_{t}^{2}-\frac{2}{p} f_{t} \leq & \frac{C}{p^{2} t}+\frac{8 n}{p^{2}} \bar{K}+\frac{8 n}{p^{2}} \sqrt{\frac{2 n}{p(2-p)} K_{1}} \\
& +\frac{4 n}{p(2-p)}\left(K_{1}+K_{3}+K_{4}\right)+\frac{1}{p^{2}} \sqrt{8 n K_{4}}
\end{aligned}
$$

on $M \times[0, T]$. For any two points $\left(x_{1}, t_{1}\right),\left(x_{2}, t_{2}\right) \in M \times[0, T]$ with $t_{1}<t_{2}$, as [1], we let $\Theta\left(x_{1}, t_{1}, x_{2}, t_{2}\right)$ the set of all the smooth paths $\gamma:\left[t_{1}, t_{2}\right] \rightarrow M$ that connect $x_{1}$ to $x_{2}$. Using the same argument in the proof of Lemma 2.10 in [1] and the inequality (4.25), for any $\gamma \in \Theta\left(x_{1}, t_{1}, x_{2}, t_{2}\right)$ we have

$$
\begin{aligned}
\frac{d}{d t} f(\gamma(t), t) & =\nabla_{t} f(\gamma(t), t) \dot{\gamma}(t)+\left.\frac{\partial}{\partial s} f(\gamma(t), s)\right|_{s=t} \\
& \geq-\left|\nabla_{t} f(\gamma(t), t)\right|_{t}|\dot{\gamma}(t)|_{t}+\frac{p}{2}\left(\left|\nabla_{t} f(\gamma(t), t)\right|_{t}^{2}-\frac{C}{p^{2} t}-A\right) \\
& \geq-\frac{1}{2 p}|\dot{\gamma}(t)|_{t}^{2}-\frac{p}{2}\left(\frac{C}{p^{2} t}+A\right),
\end{aligned}
$$

where

$$
A:=\frac{8 n}{p^{2}} \bar{K}+\frac{8 n}{p^{2}} \sqrt{\frac{2 n}{p(2-p)} K_{1}}+\frac{4 n}{p(2-p)}\left(K_{1}+K_{3}+K_{4}\right)+\frac{1}{p^{2}} \sqrt{8 n K_{4}} .
$$

Therefore, we arrive at

$$
\begin{aligned}
f\left(x_{2}, t_{2}\right)-f\left(x_{1}, t_{1}\right) & =\int_{t_{1}}^{t_{2}} \frac{d}{d t} f(\gamma(t), t) d t \\
& \geq-\frac{1}{2 p} \int_{t_{1}}^{t_{2}}|\dot{\gamma}(t)|_{g(t)}^{2} d t-\frac{p A}{2}\left(t_{2}-t_{1}\right)-\frac{C}{2 p} \ln \frac{t_{2}}{t_{1}}
\end{aligned}
$$

Corollary 4.6. Suppose that $(M, g(t))_{t \in[0, T]}$ is a solution to the geometric flow (1.1) on $M$, satisfying $-K_{1} g(t) \leq S_{g(t)} \leq K_{2} g(t),-K_{3} g(t) \leq \mathrm{Ric}_{g(t)}-S_{g(t)} \leq$ $K_{3} g(t),\left|\nabla_{g(t)} \underline{\boldsymbol{S}}_{g(t)}\right|_{g(t)} \leq K_{4}$, for some $K_{1}, K_{2}, K_{3}, K_{4}>0$, with $\bar{K}:=\max \left\{K_{1}, K_{2}\right\}$. Let $h(x, t)$ be a nonnegative function defined on $M \times[0, T]$ which is $C^{2}$ in $x$ and $C^{1}$ in $t, \Delta_{g(t)} h+h_{t}-2 C_{n, p} p \frac{\left|\nabla_{g(t)} h\right|_{g(t)}^{2}}{h} \geq 0$ on $M \times[0, T]$ (where $C_{n, p}=\frac{p}{p-1}$ if $p>1$ and $C_{n, p}=n$ if $\left.p \leq 1\right)$, and $0<p \leq \frac{2 n}{2 n-1}(n \geq 3)$. If $u$ is a positive solution of (1.2), then

$$
\begin{array}{ll}
\frac{u\left(x_{2}, t_{2}\right)}{u\left(x_{1}, t_{1}\right)} \geq & \left(\frac{t_{2}}{t_{1}}\right)^{-C / p} \exp \left[-\frac{1}{2 p} \min _{\gamma \in \Theta\left(x_{1}, t_{1}, x_{2}, t_{2}\right)} \int_{t_{1}}^{t_{2}}|\dot{\gamma}(t)|_{g(t)}^{2} d t-2 n\left(t_{2}-t_{1}\right)\right. \\
& \left.\left(\frac{1}{p} \bar{K}+\frac{2}{p} \sqrt{\frac{2 n}{p(2-p)} K_{1}}+\frac{1}{2-p}\left(K_{1}+K_{3}+K_{4}\right)+\frac{1}{p} \sqrt{2 n K_{4}}\right)\right]
\end{array}
$$

for some positive constant $C$ depending only on $n$, where $\left(x_{1}, t_{1}\right),\left(x_{2}, t_{2}\right) \in M \times$ $[0, T]$ with $t_{1}<t_{2}$.

When $K_{1}=0$, we have the following

Corollary 4.7. Suppose that $(M, g(t))_{t \in[0, T]}$ is a solution to the geometric flow (1.1) on $M$, satisfying $0 \leq S_{g(t)} \leq K_{2} g(t),-K_{3} g(t) \leq \operatorname{Ric}_{g(t)}-S_{g(t)} \leq K_{3} g(t)$, 
$\left|\nabla_{g(t)} \underline{\boldsymbol{S}}_{g(t)}\right|_{g(t)} \leq K_{4}$, for some $K_{2}, K_{3}, K_{4}>0$. Let $h(x, t)$ be a nonnegative function defined on $M \times[0, T]$ which is $C^{2}$ in $x$ and $C^{1}$ in $t, \Delta_{g(t)} h+h_{t}-$ $2 C_{n, p} p \frac{\left|\nabla_{g(t)} h\right|_{g(t)}^{2}}{h} \geq 0$ on $M \times[0, T]$ (where $C_{n, p}=\frac{p}{p-1}$ if $p>1$ and $C_{n, p}=n$ if $p \leq 1)$, and $0<p \leq \frac{2 n}{2 n-1}(n \geq 3)$. If $u$ is a positive solution of (1.2), then

$$
\begin{aligned}
\frac{u\left(x_{2}, t_{2}\right)}{u\left(x_{1}, t_{1}\right)} \geq & \left(\frac{t_{2}}{t_{1}}\right)^{-C / p} \exp \left[-\frac{1}{2 p} \min _{\gamma \in \Theta\left(x_{1}, t_{1}, x_{2}, t_{2}\right)} \int_{t_{1}}^{t_{2}}|\dot{\gamma}(t)|_{g(t)}^{2} d t\right. \\
& \left.-2 n\left(t_{2}-t_{1}\right)\left(\frac{K_{2}}{p}+\frac{K_{3}+K_{4}}{2-p}+\frac{\sqrt{2 n K_{4}}}{p}\right)\right]
\end{aligned}
$$

for some positive constant $C$ depending only on $n$, where $\left(x_{1}, t_{1}\right),\left(x_{2}, t_{2}\right) \in M \times$ $[0, T]$ with $t_{1}<t_{2}$.

Acknowledgments. The authors thank for Professor Kefeng Liu's constant guidance and help, and also referee's useful comments.

\section{REFERENCES}

[1] Bailesteanu, Mihai; Cao, Xiaodong; Pulemotov Artem. Gradient estimates for the heat equation under the Ricci flow, J. Func. Anal., 258(2010), no. 10, 3517-3542. MR2601627 (2011b: 53153)

[2] Calabi, Eugenio. An extension of E. Hopf's maximum principle with application to Riemannian geometry, Duck Math. J., 25 (1958), 45-46. MR0092069 (19, 1056e)

[3] Chen, Li; Chen, Wenyi. Gradient estimates for a nonlinear parabolic equation on complete non-compact Riemannian manifolds, Ann. Glob. Anal. Geom., 35(2009), no. 4, 397-404. MR2506242 (2010k: 35501)

[4] Li, Jiayu. Gradient estimates and Harnack inequalities for nonlinear parabolic and nonlinear elliptic equation on Riemannian manifolds, J. Funct. Anal., 100(1991), no. 2, 233-256. MR1125225 (92k: 58257)

[5] Li, Peter; Yau, Shing-Tung. On the parabolic kernel of the Schrödinger operator, Acta Math., 156(1986), no. 3-4, 153-201. MR0834612 (87f: 58156)

[6] Li, Yi;Zhu, XiaoRui. Harnack estimates for a heat-type equation under the Ricci flow, Journal of Differential Equation 260(2016), no. 4, 3270-3301.

[7] Ma, Li. Gradient estimates for a simple elliptic equation on complete non-compact Riemannian manifolds, J. Funct. Anal., 241(2006), no. 1, 374-382. MR2264255 (2007e: 53034)

[8] Ringström, Hans. The Cauchy problem in general relativity,

[9] Jun, Sun. Gradient estimates for positive solutions of the heat equation under geometric flow, Pacific Journal of Mathematics., 253(2011), no. 2, 489-510.

[10] Yang, Yunyan. Gradient estimates for a nonlinear parabolic equation on Riemannian manifolds, Proc. Amer. Math. Soc., 136(2008), no. 11, 4095-4102. MR2425752 (2009d: 58048)

[11] Zhu, Xiaorui; Li, Yi. Li-Yau estimates for a nonlinear parabolic equation on manifolds, Math. Phys. Anal. Geom., 17(2014), no. 3-4, 273-288. MR3291929

[12] Zhu, Xiaorui; Li, Yi. Hanack estimates for a nonlinear parabolic equation under Ricci flow, preprint, 2014 (submitted)

Faculty of Science, Technology and Communication (FStC), Mathematic Research Unit, Campus Belval, Universite du Luxembourg, Maison du Nombre, 6, avenue de la Fonte, L-4364, Esch-Sur-Alzette, Grand-Duchy of Luxembourg

E-mail address: yilicms@gmail.com

China Maritime Police Academy, Ningbo, 315801 China

E-mail address: zju-zxr@163.com 